\title{
High-resolution spectroscopic study of dwarf stars in the northern sky
}

\section{$\mathrm{Na}$ to $\mathrm{Zn}$ abundances in two fields with radii of 20 degrees ${ }^{\star} \star \star \star$}

\author{
Š. Mikolaitis ${ }^{1}$, A. Drazdauskas ${ }^{1}$, R. Minkevičiūtè ${ }^{1}$, E. Stonkutè ${ }^{1}$, G. Tautvaišienė ${ }^{1}$, L. Klebonas ${ }^{1,2}$, V. Bagdonas ${ }^{1}$, \\ E. Pakštiené ${ }^{1}$, and R. Janulis ${ }^{1}$

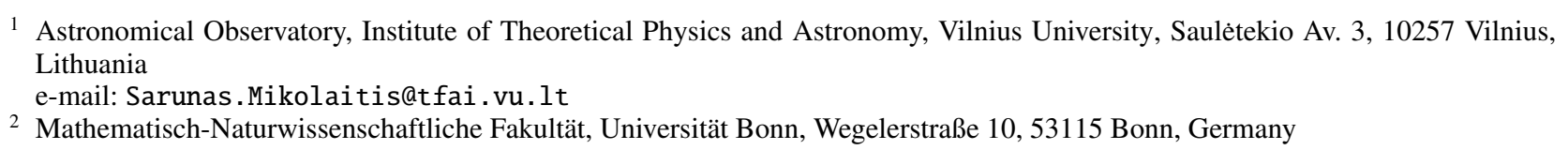

Received 31 December 2018 / Accepted 9 May 2019

\begin{abstract}
Context. New space missions, such as NASA TESS or ESA PLATO, will focus on bright stars, which have been largely ignored by modern large surveys, especially in the northern sky. Spectroscopic information is of paramount importance in characterising the stars and analysing planets possibly orbiting them, and in studying the Galactic disc evolution.

Aims. The aim of this work was to analyse all bright $(V<8 \mathrm{mag}) \mathrm{F}, \mathrm{G}$, and $\mathrm{K}$ dwarf stars using high-resolution spectra in the selected sky fields near the northern celestial pole.

Methods. The observations were carried out with the $1.65 \mathrm{~m}$ diameter telescope at the Moletai Astronomical Observatory and a fibrefed high-resolution spectrograph covering a full visible wavelength range (4000-8500 $\mathrm{A})$. The atmospheric parameters were derived using the classical equivalent width approach while the individual chemical element abundances were determined from spectral synthesis. For both tasks the one-dimensional plane-parallel LTE MARCS stellar model atmospheres were applied. The NLTE effects for the majority of elemental abundances in our sample were negligible; however, we did calculate the NLTE corrections for the potassium abundances, as they were determined from the large $7698.9 \AA$ line. For manganese and copper we have accounted for a hyperfine splitting.

Results. We determined the main atmospheric parameters, kinematic properties, orbital parameters, and stellar ages for 109 newly

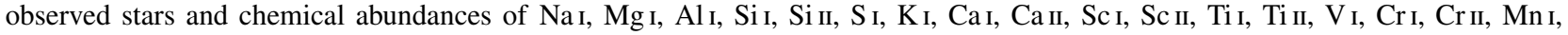
$\mathrm{Fe}_{\mathrm{I}}, \mathrm{Fe}$ II, Co I, Ni I, Cu I, and $\mathrm{Zn}$ I for $249 \mathrm{~F}, \mathrm{G}$, and $\mathrm{K}$ dwarf stars observed in the present study and in our previous study. The $[\mathrm{Mg} / \mathrm{Fe}$ I] ratio was adopted to define the thin-disc ( $\alpha$-poor) and thick-disc ( $\alpha$-rich) stars in our sample. We explored the behaviour of 21 chemical species in the $[\mathrm{El} / \mathrm{Fe} \mathrm{I}]$ versus $[\mathrm{Fe} / / \mathrm{H}]$ and $[\mathrm{El} / \mathrm{Fe} \mathrm{I}]$ versus age planes, and compared the results with the latest $\mathrm{Galactic}$ chemical evolution models. We also explored [El/Fe I] gradients according to the mean Galactocentric distances and maximum height above the Galactic plane.

Conclusions. We found that in the Galactic thin-disc [El/Fe I] ratios of $\alpha$-elements and aluminium have a positive trend with respect to age while the trend of $\mathrm{Mn}$ is clearly negative. Abundances of other species do not display significant trends. While the current theoretical models are able to reproduce the generic trends of the elements, they often seem to overestimate or underestimate the observational abundances. We found that the $\alpha$-element and zinc abundances have slightly positive or flat radial and vertical gradients, while gradients for the odd- $Z$ element $\mathrm{Na}, \mathrm{K}, \mathrm{V}$, and Mn abundances are negative.
\end{abstract}

Key words. Galaxy: disk - Galaxy: structure - Galaxy: abundances - Galaxy: stellar content - stars: abundances

\section{Introduction}

In the era of the new extra-solar planet hunting telescopes such as ESA's PLAnetary Transits and Oscillations of stars (PLATO) or NASA's Transiting Exoplanet Survey Satellite (TESS), it is very important to obtain as much information as possible on

* Full Tables 1 and A.1 are only available at the CDS via anonymous ftp to cdsarc.u-strasbg.fr (130.79.128.5) or via http: //cdsarc.u-strasbg.fr/viz-bin/qcat?]/A+A/628/A49

$\star \star$ Based on observations collected with the $1.65 \mathrm{~m}$ telescope and VUES spectrograph at the Moletai Astronomical Observatory of Institute of Theoretical Physics and Astronomy, Vilnius University, for the SPFOT survey. the stars they will be observing. These missions in particular will focus on the bright targets $(V<12 \mathrm{mag})$, that are perfectly suitable for observations with smaller ground-based telescopes. Only less than $30 \%$ of bright dwarf stars in the solar neighbourhood have been studied spectroscopically. There are more than a few spectroscopic surveys currently in progress, Gaia-ESO (Gilmore et al. 2012), GALAH (Zucker et al. 2012), APOGEE (Majewski et al. 2017), among others; however, these surveys are either based in the southern hemisphere or have other limitations such as limited wavelength coverage or a focus on fainter stars.

Coupled with the fact that the brightest stars tend to be ignored by recent surveys, we wanted to fill the existing data gap, and so have started a project to observe the bright dwarf stars in 
the northern sky (see Mikolaitis et al. 2018 for the overview of the project, hereafter Paper I).

The position of our telescope $\left(55^{\circ} 18^{\prime}\right.$ northern latitude) gives us a unique opportunity to comfortably observe stars around the northern celestial pole. Thus, for our project we employed the $1.65 \mathrm{~m}$ telescope at the Moletai Astronomical Observatory of Vilnius University together with the high-resolution Vilnius University Echelle Spectrograph (VUES) (Jurgenson et al. 2016) covering the whole visible wavelength range (4000-8500 $)$. With the data from VUES we were able to determine the main atmospheric parameters of stars $\left(T_{\text {eff }}, \log g,[\mathrm{Fe} / \mathrm{H}]\right)$ and a detailed chemical composition.

We started observations in two sky-fields with radii of $20^{\circ}$ located close to the northern celestial pole. In Paper I we presented the atmospheric parameters for 140 stars in one of the fields. In this paper we add results that include the main atmospheric parameters for stars in the second field, as well as detailed kinematic parameters and elemental abundances for stars in both fields.

It is already known that our Galaxy contains distinct disc components, notably the thin- and thick-discs. However, the exact mechanism through which these two populations formed is still debatable (e.g. Grand et al. 2018 and references therein). Studying the detailed chemical composition and kinematic properties for new samples of stars can yield deeper insights into the Galaxy formation.

The solar neighbourhood contains stars from all local Galactic components (thin- and thick-discs, and the halo); however, the majority of stars belong to the thin-disc. It is known that the thick-disc generally contains older and more metal-poor stars compared to the thin-disc, with different orbital parameters, longer scale length and larger scale height (Kordopatis et al. 2011). The most prominent feature that separates the Galactic components is the different chemical composition, the ratio of $\alpha$-elements to iron in particular (see Grisoni et al. 2017 and references therein). The solar neighbourhood has been the focus of a number of recent studies (Bensby et al. 2014; Duong et al. 2018; Frasca et al. 2018; Mishenina et al. 2017; Feuillet et al. 2018 among others). These studies differ in the stars selected, how they were selected, which elements were analysed, and how detailed the analysis was.

In this study we explore the radial and vertical abundance gradients for 21 chemical species and compare our results with the latest Galactic chemical evolution models (Romano et al. 2010; Kobayashi \& Nakasato 2011; Prantzos et al. 2018). Even though the investigated stars are not spread over large distances, as this is a study of the solar neighbourhood, if we involve the orbital parameters ( $z_{\max }$ and $\left.R_{\text {mean }}\right)$, metallicity, and age, we can investigate the original spatial distribution of the stars and study the Galactic elemental abundance gradients successfully. Our study also uses the data from the European Space Agency mission Gaia (Gaia Collaboration 2018).

This paper is organised as follows. In Sect. 2 we describe the observational data. In Sect. 3 we present the methods of analysis used to determine stellar radial velocities, kinematic properties, ages, and chemical abundances, as well as possible uncertainties. In Sect. 4 we describe the derived stellar atmospheric parameters. In Sect. 5 we discuss kinematic parameters and ages of the investigated stars. In Sect. 6 we address the determined element abundance ratios and their comparison with the Galactic evolution models. In Sect. 7 we study the radial and vertical abundance gradients in the Galactic thin-disc. In Sect. 8 we summarise the work and the results.

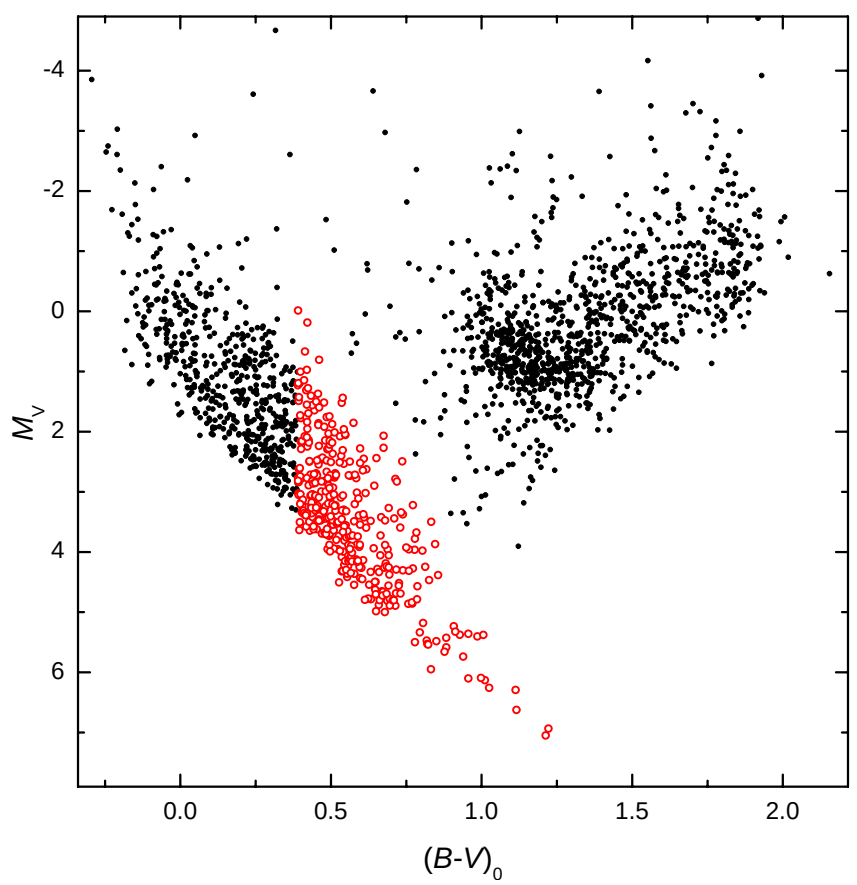

Fig. 1. Colour-magnitude diagram of stars $V<8$ mag in two fields. The FGK dwarfs (405 stars) observed in this programme are presented as red open circles.

\section{Target selection, observations, and data processing}

The methods we used for the target selection, observations, and data processing are described in Paper I. Here we only briefly summarise the most important aspects of this work. In Paper I we describe our study of the dwarf stars situated around the centre of the preliminary PLATO STEP02 field $\left(20^{\circ}\right.$ around $\alpha(2000)=161.03552^{\circ}$ and $\delta(2000)=86.60225^{\circ}$, hereafter the first field; see Rauer et al. 2014, 2016). For the second step of our project we observed another field of the northern sky of the same size that is centred on the preliminary PLATO NPF field $\left(20^{\circ}\right.$ around $\alpha(2000)=265.08003^{\circ}$ and $\delta(2000)=39.58370^{\circ}$, hereafter the second field). We constructed a colour-magnitude diagram for all selected stars in the second field. The target list consisted of 192 objects, and we observed all of them during the period of 2017-2018. We used the $1.65 \mathrm{~m}$ telescope and the high-resolution VUES spectrograph with the full visible light wavelength coverage. The primary data reduction and calibration procedures for VUES data are described in the paper by Jurgenson et al. (2016). The colour-magnitude diagram of stars from both fields is presented in Fig. 1.

\section{Method of analysis}

The method of analysis used in this study was presented in Paper I, here we only briefly recall most relevant information for the reader and the new information that is necessary.

Radial velocities were derived the same way as in Paper I using the standard cross-correlation method which also helped to identify the fast-rotating stars and the double-line binaries. Because the target list was formed using the photometric indices, the number of fast-rotating stars or double-line binaries was unknown. For the second field we found 153 stars within our data set in common with the Gaia DR2 catalogue that have the radial velocity data. In Fig. 2 we show a comparison between the 


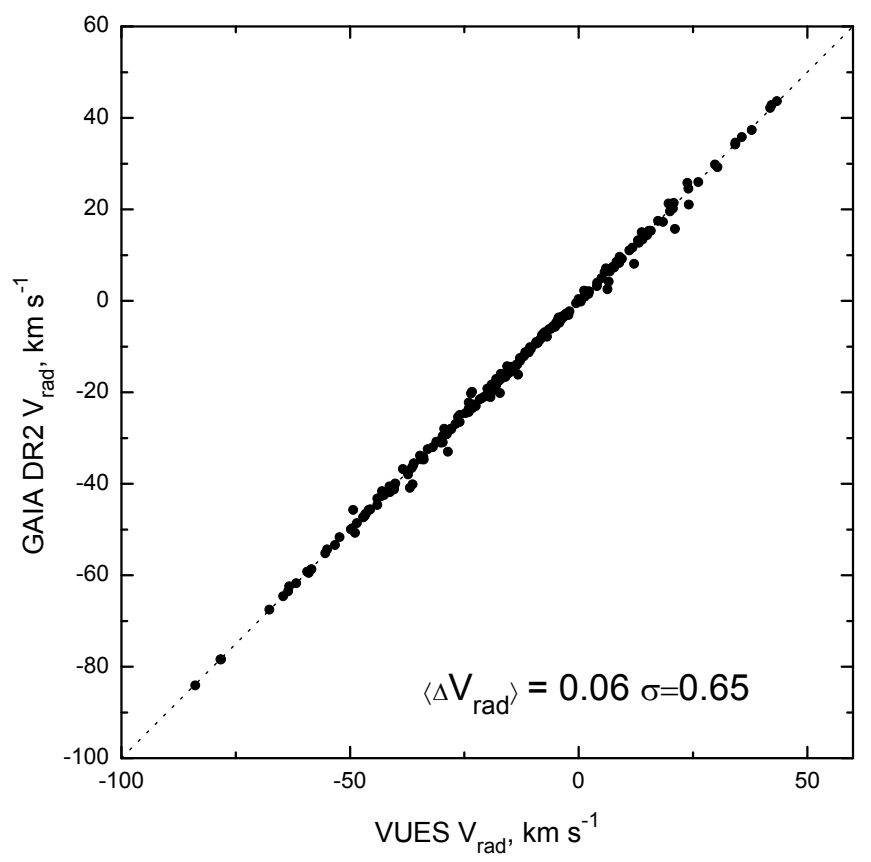

Fig. 2. Comparison of radial velocities derived in this study and in Gaia DR2 (153 stars). The dashed line with a slope of 1 is shown for comparison.

Gaia DR2 radial velocity values with those determined in this study. The mean and standard deviation of differences between the two sets is $\left\langle\Delta V_{\text {rad }}\right\rangle=0.06 \pm 0.65 \mathrm{~km} \mathrm{~s}^{-1}$. Due to the broadening and blending of the spectral lines we were not able to measure equivalent widths for 71 stars; further analysis led us to exclude 12 stars that display double-line features. Consequently, the final sample of 109 stars from the second field was used for the further analysis.

Kinematic parameters were calculated the same way as in Paper I using the python based package for galactic-dynamics calculations galpy ${ }^{1}$ by Bovy (2015). The required input data (parallaxes, proper motions and coordinates) were gathered from the Gaia DR2 catalogue (Luri et al. 2018; Katz et al. 2019; Gaia Collaboration 2016, 2018). We used the radial velocities determined in this work. We note, however, that in Paper I we used The Tycho-Gaia Astrometric Solution (TGAS) catalogue by Michalik et al. (2015); thus, in this work we decided to recompute the kinematic values for 249 stars in both fields uniformly in the light of the DR2 release of the Gaia data. We integrated the orbits for $5 \mathrm{Gyr}$ and determined the orbital parameters mentioned earlier. To account for the observational errors, for each object we performed a $1000 \mathrm{MC}$ calculations taking into account the uncertainties in the input parameters (parallaxes, proper motions, and radial velocities). We used the input values for the position and the movement of the Sun as follows: $R_{\mathrm{gc} \odot}=8 \mathrm{kpc}$, $V_{0}=220 \mathrm{~km} \mathrm{~s}^{-1}$ (Bovy et al. 2012), the distance from the Galactic plane $z_{0}=0.02 \mathrm{kpc}$ (Joshi 2007), and the local standard of rest $(U, V, W)=(11.1,12.24,7.25) \mathrm{km} \mathrm{s}^{-1}$ (Schönrich et al. 2010).

Stellar atmospheric parameters (effective temperature $T_{\text {eff }}$, surface gravity $\log g$, metallicity $\langle[\mathrm{Fe} / \mathrm{H}]\rangle^{2}$, and microturbulence velocity $v_{\mathrm{t}}$ ) were determined for 109 stars. We used the Vilnius

\footnotetext{
1 http://github.com/jobovy/galpy

2 We use the notation $[\mathrm{Fe} / \mathrm{H}]$ for the metallicity delivered using the equivalent width method and $[\mathrm{Fe} / \mathrm{I} / \mathrm{H}]$ for the neutral iron abundance computed using spectral synthesis (see Sect. 3.2).
}

Table 1. Atomic lines $(\lambda, \AA)$ used in analysis with the HFS data accounted.

\begin{tabular}{lcccc}
\hline \hline $\operatorname{Mn}_{\mathrm{I}}$ & & & & \\
$4783.4^{(B)}$ & $4823.5^{(B)}$ & $5004.9^{(B W)}$ & $5117.9^{(J)}$ & $5255.3^{(J)}$ \\
$5394.7^{(D)}$ & $5407.4^{(D e)}$ & $5420.4^{(D e)}$ & $5432.5^{(D)}$ & $5516.8^{(D e)}$ \\
$6013.5^{(H)}$ & $6016.7^{(H)}$ & $6021.8^{(H)}$ & $6440.9^{(H)}$ & \\
$\mathrm{Cu}_{\mathrm{I}}$ & & & & \\
$5105.5^{(F)}$ & $5218.2^{(\mathrm{He})}$ & $5220.1^{(\mathrm{He})}$ & $5700.2^{(\mathrm{Be})}$ & $5782.1^{(\mathrm{Be})}$ \\
\hline
\end{tabular}

Notes. The full line list is available at the CDS. References for the HFS data: ${ }^{(B)}$ Brodzinski et al. (1987), ${ }^{(B W)}$ Blackwell-Whitehead et al. (2005), ${ }^{(J)} J o h a n n e t a l .(1981),{ }^{(D)}$ Davisetal. (1971), ${ }^{(D e)}$ Dembczyńskietal.(1979), ${ }^{(H)}$ Handrichetal.(1969), ${ }^{(F)}$ Fischeretal. (1967), ${ }^{(H e)}$ Hermannetal.(1993), ${ }^{(B e)}$ Bergströmetal. (1989).

node analysis pipeline that was constantly used for the GaiaESO survey computations (Smiljanic et al. 2014). The method employs the DAOSPEC (Stetson \& Pancino 2008) software, the MOOG code (Sneden 1973), and a grid of MARCS stellar atmosphere models (Gustafsson et al. 2008). Together with the stars from Paper I, 249 stars from the two fields were selected for the chemical abundance analysis.

\subsection{Stellar ages}

To calculate the ages of our stars, we used the code UniDAM (the unified tool to estimate distances, ages and masses) by Mints \& Hekker (2017). The code uses a Bayesian approach and the PARSEC isochrones (Bressan et al. 2012). As input we use the stellar atmospheric parameters determined in this work together with the $J, H$, and $K$ magnitudes from the 2MASS survey (Skrutskie et al. 2006).

\subsection{Determination of chemical abundances}

Elemental abundances were computed using the spectral synthesis method already described in Mikolaitis et al. (2017) for the following species: $\mathrm{Na}^{3}, \mathrm{Mg}_{\text {I, }} \mathrm{Al}$ I, Si I, Si II, S I, KI, Ca I, Ca II, Sc I, Sc II, Ti i, Ti II, Vi, Cr I, Cr II, Mn I, Fe I, Fe II, Co I, $\mathrm{Ni}$ I, $\mathrm{Cu}$ I, and $\mathrm{Zn}$ I. Atomic lines were selected from the GaiaESO Survey line list (Heiter et al. 2015) and are presented in Table 1. We have also added the following molecular line lists: $\mathrm{C}_{2}$ (Brooke et al. 2013; Ram et al. 2014); CN (Sneden et al. 2014); CH (Masseron et al. 2014); SiH (Kurucz 1993); FeH (Dulick et al. 2003); $\mathrm{CaH}$ (Plez, priv. comm.); and $\mathrm{OH}, \mathrm{MgH}, \mathrm{NH}$ (Masseron, priv. comm.). The method employs MARCS (Gustafsson et al. 2008) model atmospheres and the spectral synthesis code TURBOSPECTRUM (Alvarez \& Plez 1998).

The algorithm for the abundance determination consists of (I) performing the radial velocity correction, (II) extracting the portion of the line list over the selected wavelength around the spectral line, (III) computing the synthetic spectrum, (IV) adjusting the local continuum, and (V) searching for the best synthetic spectra fit with the observed spectrum applying the minimisation of the $\chi^{2}$ parameter. The algorithm works in two steps. Firstly, it estimates the average line broadening for the $\operatorname{star}(v \sin i)$. The $v \sin i$ is determined by allowing the algorithm to iterate using two free parameters (abundance and $v \sin i$ ) and searching of the minimal $\chi^{2}$ of the fit around 139 neutral iron lines. Then the median

\footnotetext{
3 For the neutral and ionised element abundances, we use the notations
} $\left[\mathrm{El}_{\mathrm{I} / \mathrm{H}]}\right.$ and $[\mathrm{El} \mathrm{II} / \mathrm{H}]$. 
$v \sin i$ is fixed and the abundances were determined by running the algorithm again for all lines of all elements in our line list.

\subsection{Errors on atmospheric parameters}

The errors on the atmospheric patameters were estimated the same way as in Paper I.

As representative stars for our sample we chose TYC 45731916-1 and the Sun, for which we determined the scatter of results while artificially degrading their spectra to the signalto-noise ratios $(\mathrm{S} / \mathrm{Ns})$ of 25,50 , and 75 per pixel. In this way we were able to estimate the errors caused by the continuum placement and equivalent width measurement. The precision of measurement was clearly better for the best $\mathrm{S} / \mathrm{N}$ spectra. The largest errors were found for $S / N=25$ spectra $\sigma_{T_{\text {eff }}}=52 \mathrm{~K}$, $\sigma_{\log g}=0.1 \mathrm{dex}, \sigma_{[\mathrm{Fe} / \mathrm{H}]}=0.06 \mathrm{dex}, \sigma_{v_{\mathrm{t}}}=0.1 \mathrm{~km} \mathrm{~s}^{-1}$ (see Table 3 in Paper I for more details).

The previous example displays the effects of $\mathrm{S} / \mathrm{N}$ on the atmospheric parameters; however, atmospheric parameters that are based on iron line measurements also suffer from uncertainties in the atomic parameters of the used lines. All these effects create a scatter of measured iron abundances and also an error in linear regression fit that can be directly propagated to follow the uncertainties of atmospheric parameters. Therefore, the uncertainties for each of the main atmospheric parameters are provided for every star in Table A.1 (available at the CDS) and they are computed the same way as published in the description of the Vilnius node by Smiljanic et al. (2014). The median errors measured by the algorithm in the full 249 sample (both fields) are $\sigma_{\mathrm{T}_{\text {eff }}}=46 \mathrm{~K}, \sigma_{\log g}=0.3 \mathrm{dex}, \sigma_{[\mathrm{Fe} / \mathrm{H}]}=0.11 \mathrm{dex}$, and $\sigma_{v_{\mathrm{t}}}=0.27 \mathrm{~km} \mathrm{~s}^{-1}$.

\subsection{Errors on chemical abundances}

Firstly, we tested the sensitivity of the analysis method to the noise in $\chi^{2}$ fitting and continuum placement. For this we used the Monte Carlo simulations in order to add the artificial noise to a statistically significant set of spectra and followed the scatter of abundance values. Then, we studied the propagation of errors from the model atmosphere parameters to abundances:

- For the Monte Carlo simulations we used the same representative stars and their degraded spectra as in Sect. 3.3. We computed 100 abundances for each line at each $\mathrm{S} / \mathrm{N}$ value. This helped us to estimate the sensitivity of our abundances to the quality of the spectrum. These sensitivities are provided in the form of the standard deviation in Table 2.

- The evaluation of the line-to-line scatter is the way to estimate random errors. However, this error estimate is only robust when there are enough lines. We provide these error estimates for all elements in Col. 6 of Table 3, where $\sigma_{\text {scat }}^{*}$ is the median of the standard deviation for a given element.

- The uncertainties on the main atmospheric parameters that were derived in Sect. 3.3 were propagated into the errors of chemical abundances. The median errors of this type over the sample are provided in Table 3.

The final error for every element for every star in Table A.1 is a quadratic sum of effects due to uncertainty in four atmospheric parameters and the abundance scatter given by the lines.

\subsection{Testing the validity of the stellar parameters}

The metallicity value $[\mathrm{Fe} / \mathrm{H}]$ representing the ionised and neutral iron abundance was derived as the mean metallicity of a star using the equivalent width method. However, we also derived Fe I and
Table 2. Errors due to the uncertain continuum placement and line fitting as evaluated by the Monte Carlo simulations.

\begin{tabular}{|c|c|c|c|}
\hline & $S / N=25$ & $S / N=50$ & $S / N=75$ \\
\hline \multicolumn{4}{|c|}{ TYC 4573-1916-1 } \\
\hline \multicolumn{4}{|c|}{$T_{\mathrm{eff}}=6153 \mathrm{~K}, \log g=4.01,[\mathrm{Fe} / \mathrm{H}]=-0.07$} \\
\hline$[\mathrm{Na} \mathrm{I} / \mathrm{H}]$ & 0.09 & 0.08 & 0.04 \\
\hline$[\mathrm{Mg} \mathrm{I} / \mathrm{H}]$ & 0.06 & 0.06 & 0.04 \\
\hline$[\mathrm{Al} \mathrm{I} / \mathrm{H}]$ & 0.08 & 0.07 & 0.05 \\
\hline$[\mathrm{Si} \mathrm{I} / \mathrm{H}]$ & 0.06 & 0.06 & 0.06 \\
\hline$[\mathrm{Si} \mathrm{II} / \mathrm{H}]$ & 0.08 & 0.08 & 0.04 \\
\hline$[\mathrm{S} \mathrm{I} / \mathrm{H}]$ & 0.10 & 0.09 & 0.08 \\
\hline$\left[\mathrm{K}_{\mathrm{I}} / \mathrm{H}\right]$ & 0.12 & 0.10 & 0.09 \\
\hline$[\mathrm{Ca} / \mathrm{H}]$ & 0.09 & 0.09 & 0.06 \\
\hline$[\mathrm{Ca} I \mathrm{II} / \mathrm{H}]$ & 0.09 & 0.09 & 0.06 \\
\hline$[\mathrm{Sc} \mathrm{I} / \mathrm{H}]$ & 0.10 & 0.09 & 0.06 \\
\hline$[\mathrm{Sc}$ II $/ \mathrm{H}]$ & 0.11 & 0.09 & 0.07 \\
\hline$[\mathrm{Ti} \mathrm{I} / \mathrm{H}]$ & 0.08 & 0.08 & 0.06 \\
\hline [Ti II/H] & 0.08 & 0.07 & 0.04 \\
\hline$\left[\mathrm{V}_{\mathrm{I}} / \mathrm{H}\right]$ & 0.07 & 0.07 & 0.05 \\
\hline [Cr I/H] & 0.08 & 0.06 & 0.05 \\
\hline$[\mathrm{Cr}$ II $/ \mathrm{H}]$ & 0.09 & 0.07 & 0.05 \\
\hline$[\mathrm{Mn} \mathrm{I} / \mathrm{H}]$ & 0.10 & 0.08 & 0.05 \\
\hline$[\mathrm{Fe} \mathrm{I} / \mathrm{H}]$ & 0.08 & 0.06 & 0.04 \\
\hline$[\mathrm{Fe}$ II $/ \mathrm{H}]$ & 0.10 & 0.09 & 0.06 \\
\hline$[\mathrm{Co} \mathrm{I} / \mathrm{H}]$ & 0.08 & 0.07 & 0.02 \\
\hline$[\mathrm{Ni} \mathrm{I} / \mathrm{H}]$ & 0.07 & 0.06 & 0.03 \\
\hline$[\mathrm{Cu}$ I/H] & 0.08 & 0.08 & 0.06 \\
\hline$[\mathrm{Zn} \mathrm{I/H]}$ & 0.11 & 0.09 & 0.05 \\
\hline \multicolumn{4}{|c|}{$\operatorname{Sun}^{(*)}$} \\
\hline \multicolumn{4}{|c|}{$T_{\mathrm{eff}}=5779 \mathrm{~K}, \log g=4.49,[\mathrm{Fe} / \mathrm{H}]=-0.03$} \\
\hline$[\mathrm{Na} / \mathrm{H}]$ & 0.07 & 0.06 & 0.04 \\
\hline$[\mathrm{Mg} / \mathrm{H}]$ & 0.05 & 0.04 & 0.03 \\
\hline$[\mathrm{Al} \mathrm{I} / \mathrm{H}]$ & 0.05 & 0.03 & 0.01 \\
\hline$[\mathrm{Si} / \mathrm{H}]$ & 0.06 & 0.05 & 0.02 \\
\hline$[\mathrm{Si} \mathrm{II} / \mathrm{H}]$ & 0.07 & 0.04 & 0.03 \\
\hline$[\mathrm{S} \mathrm{I} / \mathrm{H}]$ & 0.10 & 0.07 & 0.07 \\
\hline$[\mathrm{K} \mathrm{I} / \mathrm{H}]$ & 0.10 & 0.09 & 0.08 \\
\hline$[\mathrm{Ca} / \mathrm{H}]$ & 0.07 & 0.04 & 0.03 \\
\hline$[\mathrm{Ca} \mathrm{II} / \mathrm{H}]$ & 0.07 & 0.06 & 0.05 \\
\hline$[\mathrm{Sc} \mathrm{I} / \mathrm{H}]$ & 0.09 & 0.07 & 0.05 \\
\hline$[\mathrm{Sc} \mathrm{II} / \mathrm{H}]$ & 0.11 & 0.10 & 0.07 \\
\hline$[\mathrm{Ti} \mathrm{I} / \mathrm{H}]$ & 0.07 & 0.07 & 0.05 \\
\hline [Ti II/H] & 0.08 & 0.08 & 0.05 \\
\hline$\left[\mathrm{V}_{\mathrm{I}} / \mathrm{H}\right]$ & 0.06 & 0.03 & 0.01 \\
\hline$[\mathrm{Cr} \mathrm{I} / \mathrm{H}]$ & 0.05 & 0.03 & 0.02 \\
\hline$[\mathrm{Cr}$ II $/ \mathrm{H}]$ & 0.08 & 0.07 & 0.07 \\
\hline$[\mathrm{Mn} \mathrm{I} / \mathrm{H}]$ & 0.06 & 0.03 & 0.03 \\
\hline$[\mathrm{Fe} \mathrm{I} / \mathrm{H}]$ & 0.05 & 0.03 & 0.02 \\
\hline$[\mathrm{Fe}$ II $/ \mathrm{H}]$ & 0.09 & 0.08 & 0.06 \\
\hline$[\mathrm{Co} \mathrm{I} / \mathrm{H}]$ & 0.08 & 0.07 & 0.05 \\
\hline$[\mathrm{Ni} / \mathrm{I} / \mathrm{H}]$ & 0.05 & 0.05 & 0.02 \\
\hline$[\mathrm{Cu} \mathrm{I} / \mathrm{H}]$ & 0.07 & 0.05 & 0.04 \\
\hline [Zn I/H] & 0.08 & 0.07 & 0.04 \\
\hline
\end{tabular}

Notes. ${ }^{(*)}$ Solar atmospheric parameters derived with our method.

Fe II using spectral synthesis method. Since the iron abundances were derived using different and slightly independent ways it is important to check if they are consistent. We provide this comparison in the first row of Fig. 3. The bias of $[\mathrm{Fe} \mathrm{I} / \mathrm{Fe}]$ is $+0.03 \mathrm{dex}$ with a scatter $\sigma=0.06$ dex. We did not find any noticeable systematic effects with respect to $T_{\text {eff }}, \log g$, or $[\mathrm{Fe} / \mathrm{H}]$. 
Table 3. Median effects on the derived $[\mathrm{El} / \mathrm{Fe}]$ ratios resulting from the atmospheric parameter uncertainties for the sample stars.

\begin{tabular}{|c|c|c|c|c|c|c|c|c|}
\hline $\mathrm{El}$ & $\begin{array}{c}\Delta T_{\text {eff }} \\
\mathrm{K}\end{array}$ & $\Delta \log g$ & $\Delta[\mathrm{Fe} / \mathrm{H}]$ & $\begin{array}{c}\Delta v_{\mathrm{t}} \\
\mathrm{km} \mathrm{s}^{-1}\end{array}$ & $\sigma_{\text {scat }}(1)$ & $N_{\max }(2)$ & $\sigma_{\text {total }}\left[\frac{\mathrm{x}}{\mathrm{Fe}}\right]$ & $\sigma_{\text {all }\left[\frac{\mathrm{X}}{\mathrm{Fe}}\right]}{ }^{(4)}$ \\
\hline $\mathrm{Na}$ I & 0.01 & 0.04 & 0.02 & 0.02 & 0.03 & 4 & 0.05 & 0.06 \\
\hline $\mathrm{Mg}_{\mathrm{I}}$ & 0.01 & 0.06 & 0.02 & 0.03 & 0.07 & 5 & 0.07 & 0.11 \\
\hline $\mathrm{Al}$ & 0.01 & 0.02 & 0.02 & 0.03 & 0.04 & 5 & 0.03 & 0.06 \\
\hline Si I & 0.01 & 0.01 & 0.02 & 0.03 & 0.03 & 14 & 0.03 & 0.04 \\
\hline Si II & 0.02 & 0.05 & 0.02 & 0.03 & 0.07 & 7 & 0.06 & 0.10 \\
\hline$S_{\text {I }}$ & 0.04 & 0.08 & 0.02 & 0.05 & 0.09 & 4 & 0.10 & 0.14 \\
\hline $\mathrm{K}_{\mathrm{I}}$ & 0.03 & 0.07 & 0.03 & 0.04 & 0.06 & 1 & 0.10 & 0.13 \\
\hline $\mathrm{Ca}_{\mathrm{I}}$ & 0.02 & 0.07 & 0.02 & 0.03 & 0.05 & 31 & 0.08 & 0.09 \\
\hline Ca II & 0.02 & 0.06 & 0.03 & 0.04 & 0.06 & 7 & 0.08 & 0.10 \\
\hline $\mathrm{Sc}_{\mathrm{I}}$ & 0.04 & 0.03 & 0.02 & 0.03 & 0.08 & 7 & 0.06 & 0.11 \\
\hline Sc II & 0.01 & 0.09 & 0.02 & 0.04 & 0.04 & 12 & 0.10 & 0.11 \\
\hline Ti I & 0.04 & 0.04 & 0.02 & 0.03 & 0.04 & 81 & 0.07 & 0.08 \\
\hline Ti II & 0.02 & 0.08 & 0.03 & 0.04 & 0.04 & 19 & 0.09 & 0.10 \\
\hline $\mathrm{V}_{\mathrm{I}}$ & 0.03 & 0.02 & 0.02 & 0.03 & 0.05 & 8 & 0.04 & 0.06 \\
\hline Cri & 0.02 & 0.03 & 0.02 & 0.03 & 0.04 & 21 & 0.04 & 0.06 \\
\hline Cr II & 0.02 & 0.08 & 0.02 & 0.04 & 0.04 & 2 & 0.09 & 0.11 \\
\hline Mn I & 0.03 & 0.03 & 0.03 & 0.03 & 0.05 & 14 & 0.06 & 0.08 \\
\hline $\mathrm{Fe}_{\mathrm{I}}$ & 0.02 & 0.03 & 0.02 & 0.03 & 0.05 & 138 & 0.05 & 0.05 \\
\hline $\mathrm{Fe}_{\text {II }}$ & 0.02 & 0.09 & 0.02 & 0.05 & 0.05 & 11 & 0.10 & 0.11 \\
\hline Co I & 0.02 & 0.01 & 0.02 & 0.03 & 0.05 & 7 & 0.03 & 0.06 \\
\hline $\mathrm{Ni}$ I & 0.02 & 0.02 & 0.02 & 0.03 & 0.03 & 30 & 0.04 & 0.06 \\
\hline $\mathrm{Cu}$ & 0.03 & 0.02 & 0.03 & 0.03 & 0.05 & 6 & 0.05 & 0.07 \\
\hline $\mathrm{Zn}_{\mathrm{I}}$ & 0.01 & 0.02 & 0.02 & 0.03 & 0.09 & 3 & 0.04 & 0.10 \\
\hline
\end{tabular}

Notes. ${ }^{(1)} \sigma_{\text {scat }}$ stands for median line-to-line scatter; ${ }^{(2)} N_{\text {max }}$ presents the number of lines investigated; ${ }^{(3)} \sigma_{\text {total([X/Fe]) }}$ stands for the median of the quadratic sum of all four effects on $[\mathrm{El} / \mathrm{Fe}]$ ratios; ${ }^{(4)} \sigma_{\text {all }(\mathrm{X} / \mathrm{Fe}])}$ is the median combined effect of $\sigma_{\text {total([El/Fe] })}$ and the line-to-line scatter $\sigma_{\text {scat }}$.

The comparison of neutral and ionised elemental abundances are used to check the ionisation equilibrium. In Fig. 3 we provide similar comparisons for [Fe I/Fe II], [Si I/Si II], [Ca I/Ca II], [Sc I/Sc II], [Ti I/TiII], and [CrI/CrII]. The bias is mostly quite small, from $-0.03 \mathrm{dex}$ for $[\mathrm{Ca} / \mathrm{Ca}$ II] $(\sigma=0.06 \mathrm{dex})$ to $0.04 \mathrm{dex}$ for $[\mathrm{Ti}$ I/Ti II] $(\sigma=0.06 \mathrm{dex})$, whereas the largest scatter is $\sigma=0.07 \mathrm{dex}$ for $[\mathrm{Sc} \mathrm{I} / \mathrm{Sc}$ II $]$. We did not find noticeable systematic effects with respect to $\log g$ or $[\mathrm{Fe} / \mathrm{H}]$. However, [Ti I/Ti II] and [Sc I/Sc II] versus $T_{\text {eff }}$ distributions show some small dependences. Therefore, we conclude that the noticed biases, scatter, and dependences are relatively small and should not affect our study.

\subsection{NLTE effects on abundances}

There is evidence that silicon and titanium can be affected by non-local thermodynamic equilibrium (NLTE) effects (e.g. Bergemann 2011; Bergemann et al. 2013). The NLTE effects for silicon abundances are mostly noticeable in supergiant stars (see Bergemann et al. 2013); however, Bergemann (2011) and Zhao et al. (2016) recommend taking NLTE into account for lines of ionised titanium in spectra of giant stars as well. Thus, as the NLTE effects for the Ti lines are largest in metal-poor and giant stars (Zhao et al. 2016), it should be safe to use the classical LTE approach to compute $\alpha$-element abundances for our sample stars, which are mostly metal-rich dwarfs. According to the results by Zhao et al. (2016), Ca and Sc should only have larger NLTE-LTE departures in the low-metallicity regime $([\mathrm{Fe} / \mathrm{H}]<-1.5 \mathrm{dex})$, which should not impact our sample as well. Magnesium abundances also have very low NLTE departures for the majority of the lines that we used (Zhao et al. 2016; Alexeeva et al. 2018).
Zhao et al. (2016) showed that sodium abundances are notably sensitive to NLTE effects for metallicities lower than $\approx-1.4$ dex and are almost insignificant for metal-rich dwarfs. The aluminium abundances can be affected by NLTE (Baumueller \& Gehren 1997; Gehren et al. 2004, 2006; Andrievsky et al. 2008). Baumueller \& Gehren (1997) calculated the LTE and NLTE aluminium abundances using the spectral lines at $\sim 8772 \AA$, which we used in our analysis as well. For stars that are similar to the ones analysed in this work, the NLTE abundances are up to 0.07 dex higher than the LTE values, thus the corrections would be similar to the errors arising from the uncertainties in parameters and are quite small. Zhao et al. (2016) computed the NLTE and LTE abundances for aluminium lines at $6696 \AA$ and $6698 \AA$ as well, and showed that there are differences of up to $\approx 0.1$ dex. Considering that the differences are quite small, the NLTE-LTE departures should not be the main source of dispersion for $\mathrm{Al}$ in this metallicity range.

Korotin et al. (2017) calculated the NLTE corrections for sulphur lines used by the Gaia-ESO survey and showed that they generally do not exceed 0.07 dex for dwarfs. It was shown by Zhao et al. (2016) and Takeda et al. (2002), who computed NLTE and LTE abundances for the most prominent $\mathrm{K}_{\mathrm{I}} 7698 \AA$ A line for $\mathrm{F}$ and $\mathrm{G}$ dwarfs, that potassium has much more noticeable NLTE effects that range from approximately -0.3 to $-0.6 \mathrm{dex}$ in the metallicity regime, which is similar to the values in our sample. In this study we applied the NLTE corrections from Takeda et al. $(2002,2009)^{4}$ for the $7698 \AA$ potassium line and report the NLTE abundance results.

\footnotetext{
4 http://www2 .nao.ac.jp/ takedayi/potassium_nonlte
} 


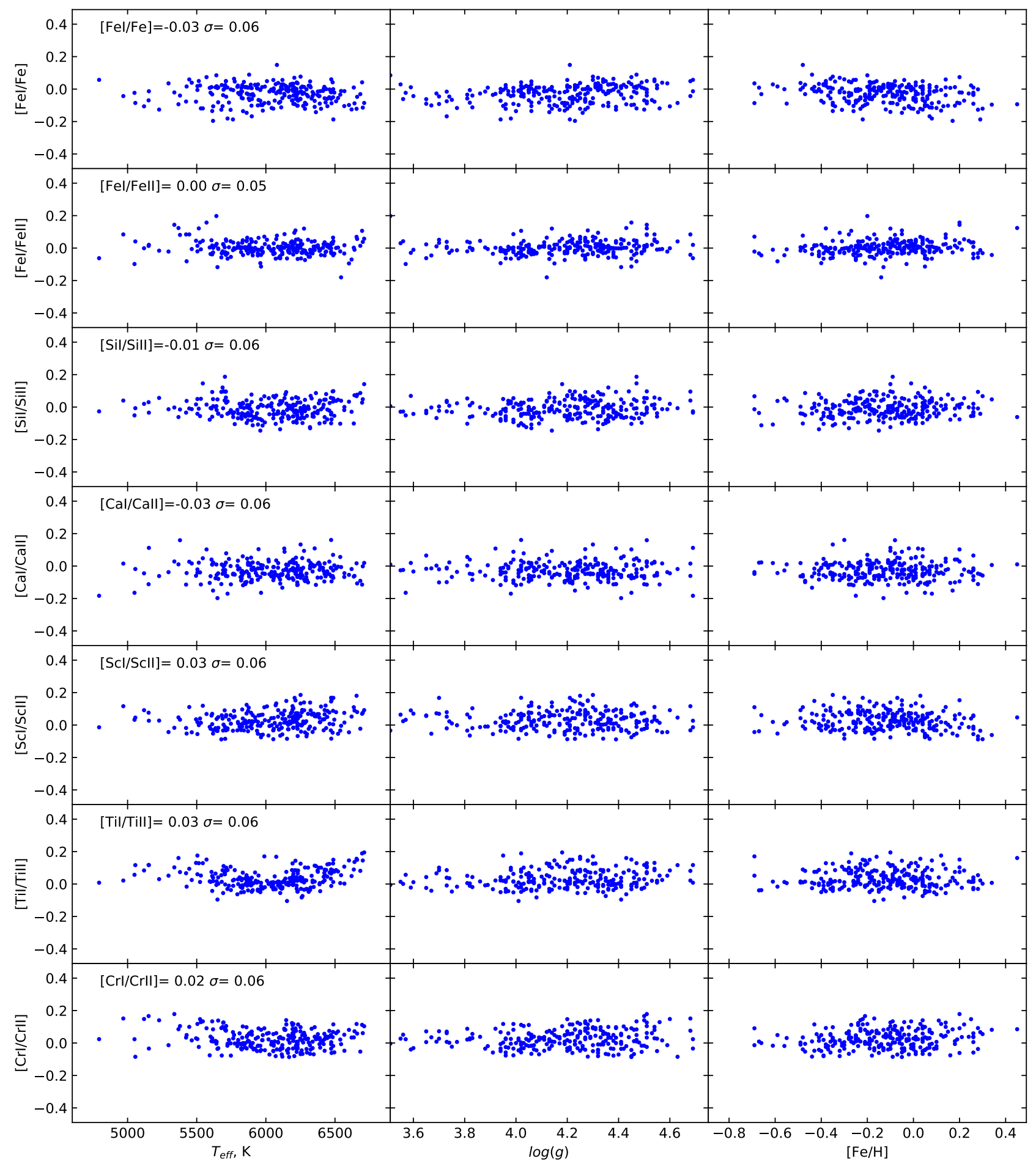

Fig. 3. Differences between the mean abundances of $\mathrm{Si}, \mathrm{Ca}, \mathrm{Ti}$, and $\mathrm{Cr}$ and their ionised counterparts as a function of the atmospheric parameters.

We note that there should be some NLTE effects influencing the determined manganese abundances. Some NLTE deviations have already been studied in previous works. Mishenina et al. (2015), who studied stars similar to those in our sample, calculated the average LTE-NLTE abundance variations of $0.01 \pm 0.04$ and $0.02 \pm 0.04$ dex for the thin- and thick-disc stars, respectively. Bergemann \& Gehren (2008) also showed that the NLTE corrections for manganese in metal-rich dwarfs should be around 0.05 dex. Battistini \& Bensby (2015) reported the NLTE abundance corrections of $0.059 \pm 0.046$ dex for stars similar to ours $([\mathrm{Fe} / \mathrm{H}]>-0.7 \mathrm{dex})$. They reported the $0.069 \pm 0.036 \mathrm{dex}$ NLTE abundance corrections for cobalt as well. Vanadium, chromium, and nickel were barely studied using the NLTE approach. The NLTE correction for the solar abundances of $\mathrm{Ni}$ and $\mathrm{Cr}$ are negligible, whereas the corrections for vanadium could reach $\approx 0.1$ dex (see Scott et al. 2015; Sneden et al. 2016). Therefore, the classical LTE determinations for the iron-peak element abundances ( $\mathrm{V}, \mathrm{Cr}, \mathrm{Co}, \mathrm{Ni}, \mathrm{Mn}$ ) can be accepted within the given uncertainty of 0.1 dex.

The NLTE effects for the $\mathrm{Cu}$ abundances should not be significant to our results. Shi et al. (2014) and Yan et al. (2016) have shown that the NLTE-LTE corrections for $\mathrm{Cu}$ abundances 


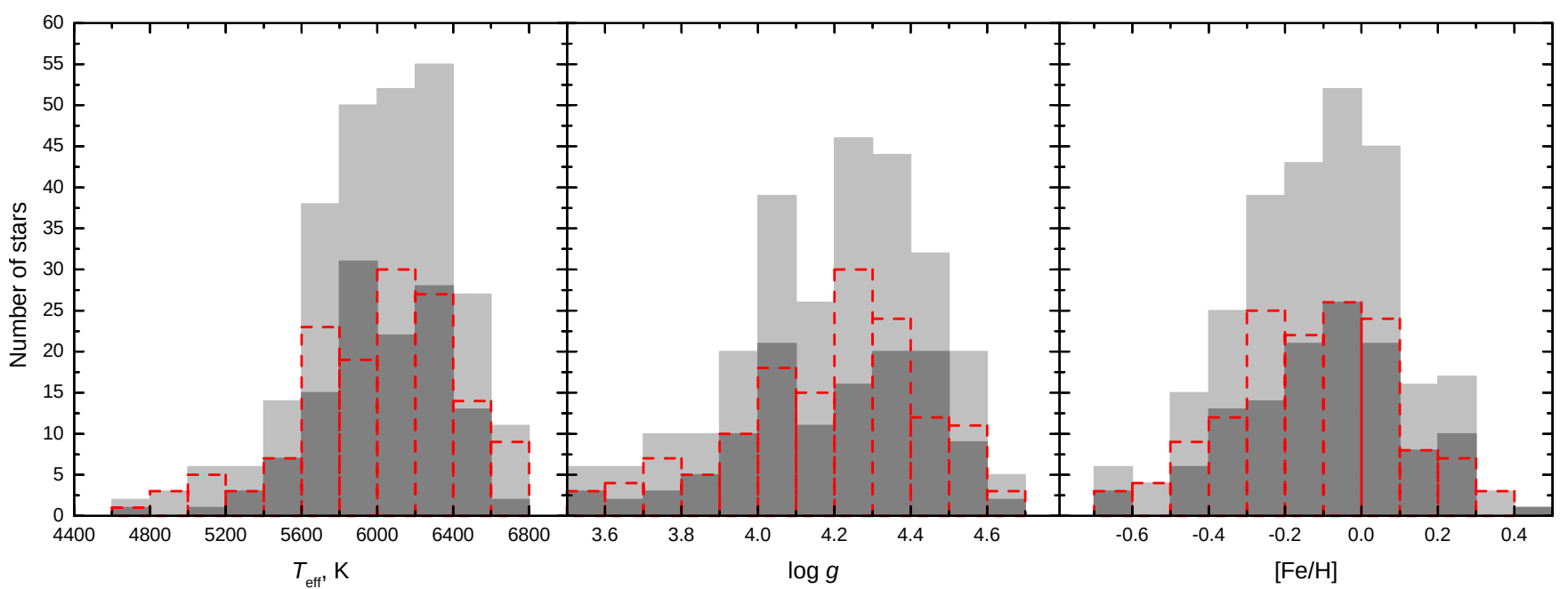

Fig. 4. Histograms of the determined spectroscopic parameters $\left(T_{\text {eff }}, \log g\right.$, and $\left.[\mathrm{Fe} / \mathrm{H}]\right)$ for all stars in our sample, the dark grey columns are for the NPF field, the red dashed lines are for the STEP 02 field, and the light grey columns are for both fields together.

in stars similar to the values in our sample are not very large (up to $0.1 \mathrm{dex}$ for $[\mathrm{Fe} / \mathrm{H}] \approx-1.0$ ). Moreover, Zhao et al. (2016) have shown that the $\mathrm{Cu}$ NLTE effects are not very significant in a star sample similar to ours. The Zn I $6362 \AA$ line is affected by the broad auto-ionisation absorption feature of $\mathrm{Ca}$ I as identified by Mitchell \& Mohler (1965). Chen et al. (2004) showed that diferentially with respect to the Sun, the largest effect of the mentioned $\mathrm{Ca}$ I feature would only be about 0.02 dex. That is a significantly smaller value than our typical error of $\mathrm{Zn}$ abundance, thus it has been neglected in the present analysis.

\section{Atmospheric parameters}

In this study, we determined atmospheric parameters for 109 stars of the NPF field to complement the previously determined 140 results of the STEP 02 field. The stellar parameters are provided in Table A.1. Distributions of the determined atmospheric parameters for the stars in both fields are shown in Fig. 4. They are quite similar: the effective temperatures of the stars range from $T_{\text {eff }} \approx 4700 \mathrm{~K}$ to $6900 \mathrm{~K}$ (Fig. 4a) with a peak at $\approx 6000 \mathrm{~K} ; \log g$ values range from $\approx 3.5$ to 4.7 (Fig. $4 \mathrm{~b}$ ); and $[\mathrm{Fe} / \mathrm{H}]$ values range from $\approx-0.7$ to $+0.4 \mathrm{dex}$ (Fig. 4 ), where the majority of the stars have approximately solar $[\mathrm{Fe} / \mathrm{H}]$. In addition, we plot the $\left(T_{\text {eff }}, \log g\right)$ diagram with colour-coded metallicity in Fig. 5. The stellar evolutionary tracks by Girardi et al. (2000) are plotted in the background. Their masses are between 0.7 and $1.9 M_{\odot}$ and the initial metallicity $Z_{\text {ini }}=0.019$.

In Paper I we compared our results with other studies. The comparison with a number of results from other spectroscopic studies (27\% of stars in common) showed rather good compatibility $\left(\left\langle\Delta T_{\text {eff }}\right\rangle=7 \pm 73 \mathrm{~K},\langle\Delta \log g\rangle=0.02 \pm 0.09\right.$, and $\langle\Delta[\mathrm{Fe} / \mathrm{H}]\rangle=$ $-0.02 \pm 0.08 \mathrm{dex})$. The scatter of our results was slightly higher $\left(\left\langle\Delta T_{\text {eff }}\right\rangle=14 \pm 125 \mathrm{~K},\langle\Delta \log g\rangle=0.02 \pm 0.16\right.$, and $\langle\Delta[\mathrm{Fe} / \mathrm{H}]\rangle=$ $-0.02 \pm 0.09 \mathrm{dex})$ with respect to the values from the photometric study by Casagrande et al. (2011). Additionally, we show a comparison of our effective temperatures (both fields) with the Gaia DR2 results (245 stars in common) in Fig. 6.

\section{Kinematic parameters and ages}

As we note in Sect. 3 of this work, we determined stellar kinematic parameters for both fields using the new Gaia DR2 data. In

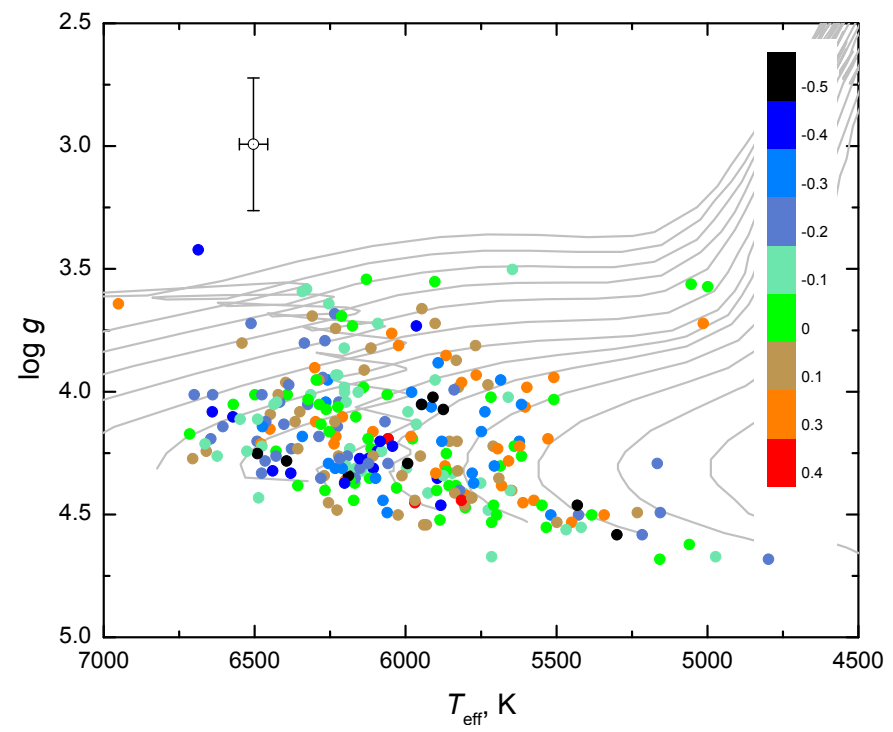

Fig. 5. Temperature-gravity diagram of 249 stars (dots) of the investigated fields with colour-coded metallicity. Evolutionary tracks with masses between 0.7 and $1.9 M_{\odot}$ and $Z_{\text {ini }}=0.019$ by Girardi et al. (2000) are plotted as grey solid lines.

Fig. 7 we present the kinematic distribution of our sample stars (249 objects).

A Toomre diagram is a way to understand the sample in the context of Galactic populations. It helps in the study of stellar combined vertical and radial kinetic energies versus the rotational energy (Bensby et al. 2014). The stars with a lower combined velocity $v_{\text {tot }}<50 \mathrm{~km} \mathrm{~s}^{-1}\left(v_{\text {tot }}=\left(U_{\mathrm{LSR}}+V_{\mathrm{LSR}}+W_{\mathrm{LSR}}\right)^{1 / 2)}\right)$ are most probably the thin-disc star, whereas those with $v_{\text {tot }}>$ $50 \mathrm{~km} \mathrm{~s}^{-1}$ should belong to the thick-disc (see Fig. 7 panel a).

Another way to disentangle the sample is to study the thickto-thin disc probability ratios $(T D / D)$. The method that we used to compute the $T D / D$ is described in Bensby et al. (2003, 2005, 2014), where it was advised to classify a star as thick-disc by requiring it to have a probability at least two times larger than that of being a thin-disc star. Thus, all stars with $T D / D>2.0$ should be the thick-disc candidates and $T D / D<0.5$ should be thin-disc candidates. 


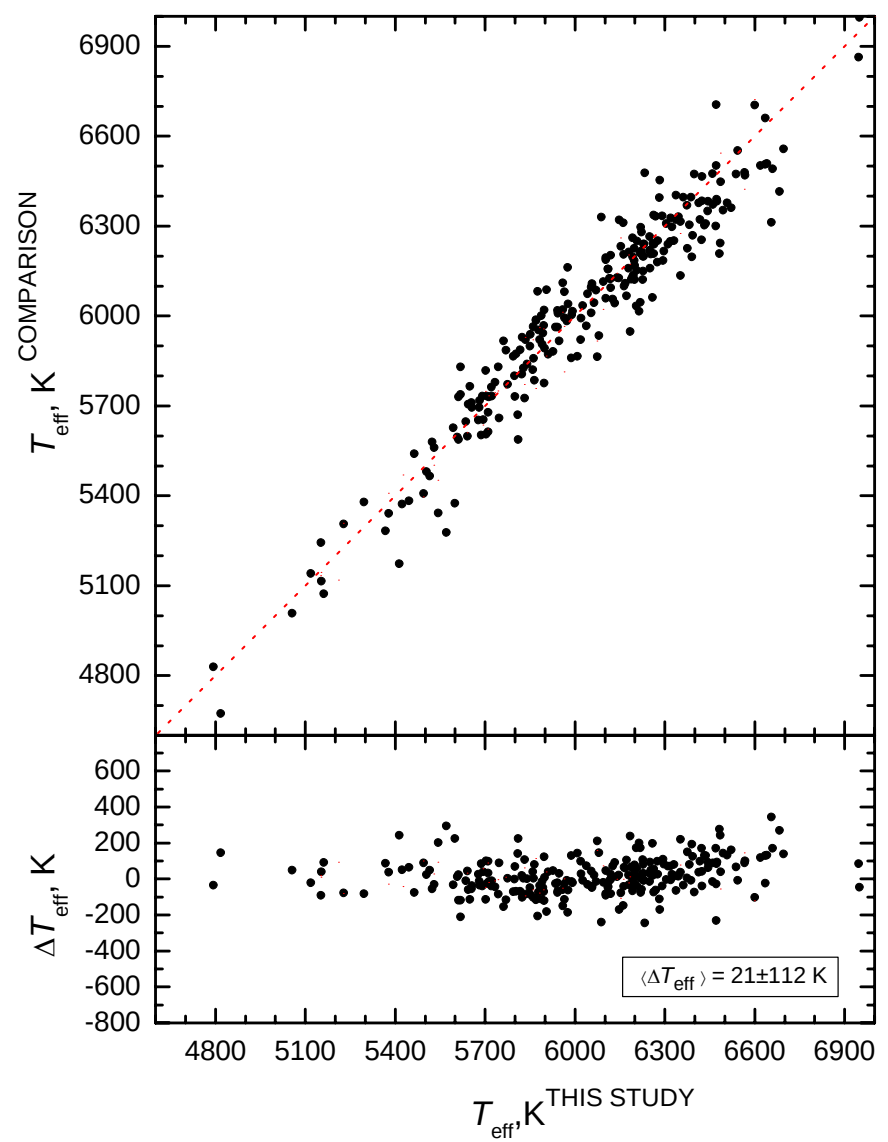

Fig. 6. Comparison between $T_{\text {eff }}$ values of our study with values from the Gaia DR2 (245 stars, black dots). The red dashed lines with a slope of 1 are shown for comparison.

Following the first criterion we selected 172 thin-disc and 25 thick-disc stars, and 57 should be transition stars between the thin- and thick-discs. The second criterion indicated only three thick-disc stars and only one star would be intermediate.

As is seen from Fig. 8, the ages of the stars in our sample range from 1 to $10 \mathrm{Gyr}$; the majority have ages close to solar, about 4 Gyr. Haywood et al. (2013) suggest that stars younger than $\approx 8 \mathrm{Gyr}$ should be from the thin-disc population and those older than $\approx 8 \mathrm{Gyr}$ are either from the thick-disc population or the metal-poor thin-disc. Our sample is composed of mostly younger generation stars: 238 stars are younger than $\approx 8 \mathrm{Gyr}$ and only 12 stars are $\approx 8$ Gyr to $\approx 10$ Gyr old. However, we have only two stars that have probability ratio $T D / D$ close to $100 \%$ and both are found in the older part of panel d in Fig. 7.

The chemical division of thin- and thick-disc populations that is based on $\alpha$-to-iron abundance ratios can be also useful. We used this method in our work, as discussed in the next section.

\section{Elemental abundance ratios and comparison with models}

It is important to study the chemical abundance ratios and their patterns in spatial and temporal context as they are fingerprints of the Galactic chemical evolution history. In Figs. 9 and A.1 we show the ratios $[\mathrm{El} / \mathrm{Fe} \mathrm{I}]$ versus $[\mathrm{Fe} \mathrm{I} / \mathrm{H}]$. This way of representing elemental abundance ratios is commonly used to chemically disentangle Galactic substructures (thin-disc, thick-disc, and halo) which have different chemical properties (e.g. Fuhrmann 2004; Neves et al. 2009; Bensby et al. 2003, 2014; Adibekyan et al.
2012; Mikolaitis et al. 2017). This so-called chemical tagging is one of the ways to separate thin- and thick-discs. In particular, it has been shown that the chemical distinction of the thinand thick-discs is possible by using $[\alpha / \mathrm{Fe}]$ ratios and it has been used in a number of studies (e.g. Fuhrmann 1998; Recio-Blanco et al. 2014; Rojas-Arriagada et al. 2016; Mikolaitis et al. 2014, 2017). In Mikolaitis et al. (2014, 2017) we already discussed that $[\mathrm{Mg} / \mathrm{Fe}]$ is one of the best indicators to define the Galactic disc components. We used the same method as in Mikolaitis et al. $(2014,2017)$ to chemically tag 223 stars as thin ( $\alpha$-rich) disc members and 26 stars as thick ( $\alpha$-poor) disc members in our sample. In the $\left[\mathrm{Mg}_{\mathrm{I}} / \mathrm{Fe}_{\mathrm{I}}\right]$ versus $\left[\mathrm{Fe}_{\mathrm{I}} / \mathrm{H}\right]$ plot in Fig. A.1 we display both chemically defined populations in different colours: blue for the thin-disc and red for the thick-disc stars. We keep this colouring style throughout the entire paper for the two discs. We also show the chemical abundance ratio variations in relation to the age, $R_{\text {mean }},\left|z_{\max }\right|$ in Figs. 10 , A.2, 11, and 12, where we provide the numerical evaluation of the gradients as well.

Moreover, chemical abundance ratios can be used as a test for the Galactic chemical evolution (GCE) models. A good GCE model would have to explain the dynamical properties of the disc, and also its chemical element distributions according to metallicity and age or radial and vertical gradients. The GCE models differ in their internal formalism, input parameters, and various assumptions (see Nomoto et al. 2013). For example, the different input parameter combinations that can be used in GCE models can include different types of supernovae, different chemical element yields which can be used to constrain the nucleosynthesis scenarios, and nucleosynthesis timescales.

Our study is based on the sample of stars that are very well confined in the solar vicinity. As can be seen from the second panel in Fig. 7, all stars are less than $0.42 \mathrm{kpc}$ from the Sun, and most are closer than $0.15 \mathrm{kpc}$. We compare several recent GCE models with our determined abundance ratios $[\mathrm{El} / \mathrm{Fe} \mathrm{I}]$ in relation to $[\mathrm{Fe} \mathrm{I} / \mathrm{H}]$ and age. For this purpose we selected GCE models developed by Romano et al. (2010), Kobayashi \& Nakasato (2011), and Prantzos et al. (2018). These studies explicitly provide the GCE in the context of metallicity and age for all or majority of species analysed in this work. In the case of Romano et al. (2010), we took their 1st (hereafter R1) and 15th (hereafter R15) models, which represent two completely different model inputs. Model R1 is based on adopted yields from Woosley \& Weaver (1995) case A and van den Hoek \& Groenewegen (1997). Model R15 is computed using the yields from Kobayashi et al. (2006) $\left(\varepsilon_{\mathrm{HN}}=1\right)$, the Geneva-group (Ekström et al. 2008) presupernovae yields computed with rotation and mass loss and the yields from low- and intermediate-mass stars (Karakas 2010). From the Kobayashi \& Nakasato (2011) we took the solar neighbourhood thin-disc model (hereafter $\mathrm{K}_{\text {thin }}$ - magenta line) with the recomputed yields and updated star formation rates (compared to the Kobayashi et al. 2006 model). Finally, we plotted the baseline model (hereafter PB) from Prantzos et al. (2018), which for the first time includes, among other factors, the combined effect of metallicity, mass loss, and rotation. We show cases where all models completely fail to represent the data in Figs. 9 and 10, whereas all plots are presented in Figs. A.1 and A.2. We also discuss the abundances of elements for the 249 stars in our sample and compare them with the selected models.

\section{1. $\mathrm{Mg}, \mathrm{Si}, \mathrm{Ca}, \mathrm{Sc}$, and $\mathrm{Ti}$}

We observe distinguishable trends for $\alpha$-elements (magnesium, silicon, calcium, scandium, and titanium) of thick- and thindisc members with higher and lower $\alpha$-element abundances, 

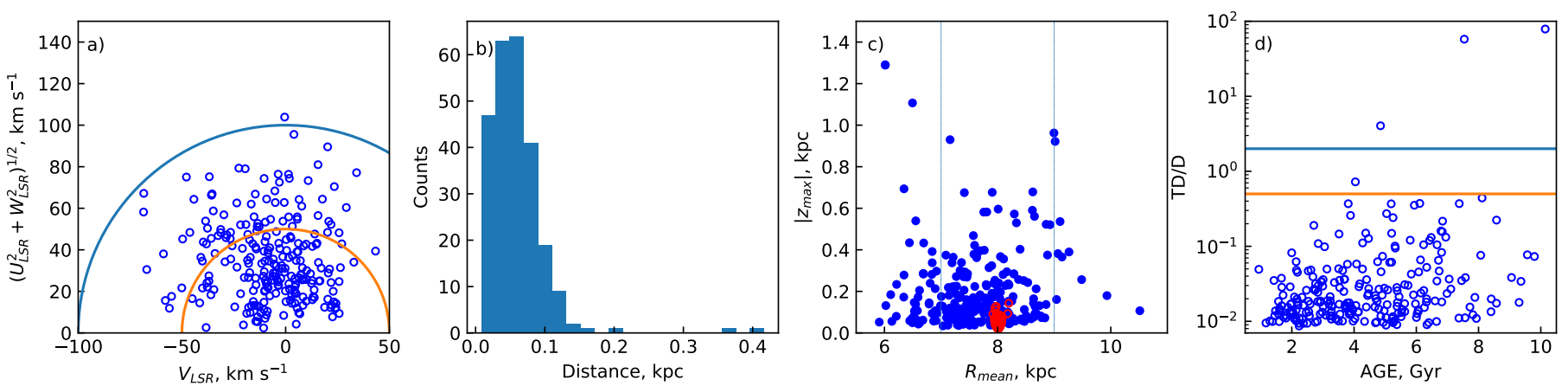

Fig. 7. Kinematic parameters: $(a)$ Toomre diagram of sample data with lines that show constant values of the total space velocity $\left(v_{\text {tot }}=\left(U_{\text {LSR }}+\right.\right.$ $\left.\left.V_{\mathrm{LSR}}+W_{\mathrm{LSR}}\right)^{1 / 2)}\right)$ at 50 and $100 \mathrm{~km} \mathrm{~s}^{-1} ;(b)$ histogram of distances of sample stars; $(c)$ distribution of sample stars in $z_{\text {max }}$ vs. $R_{\text {mean }}$ plane, where the two vertical dashed lines delimit the solar neighbourhood $7<R_{\mathrm{gc}}<9 \mathrm{kpc}$ and red circles are current positions $(|z|$ vs. $R$ ) for comparison; $(d)$ kinematical thick-to-thin disc probability ratios $(\mathrm{TD} / \mathrm{D})$ vs. age for the sample stars, where the upper and lower lines mark $T D / D=2.0$ and $T D / D=0.5$, respectively.

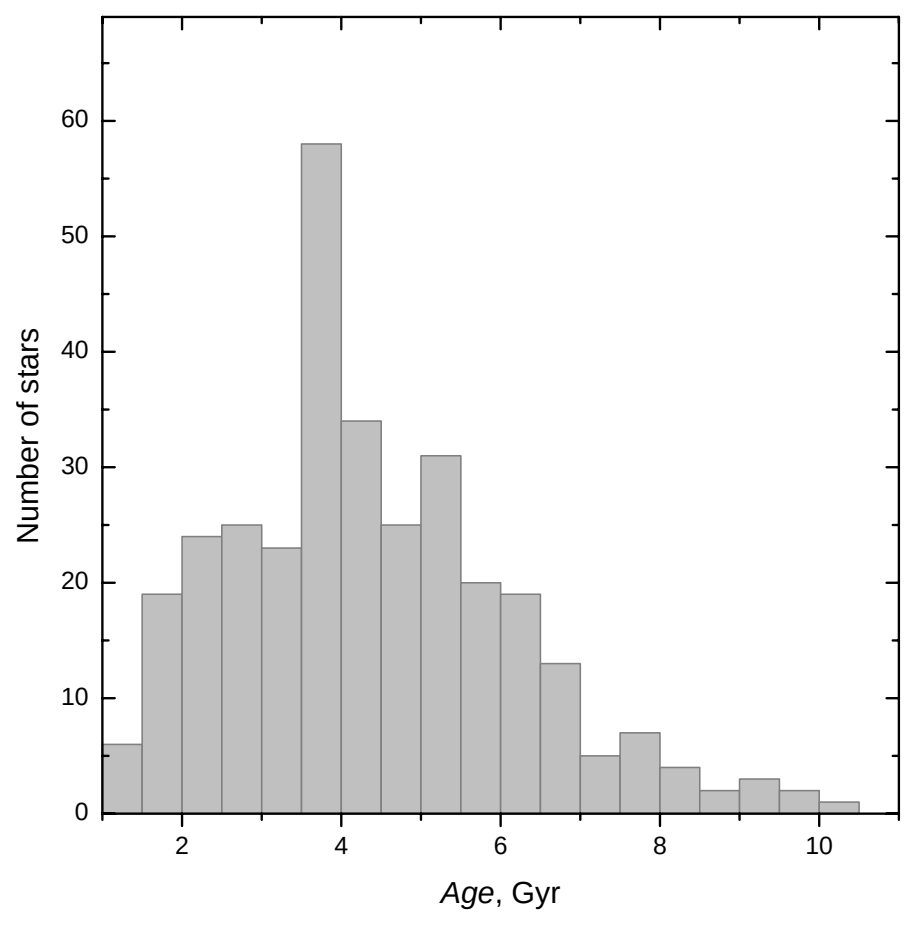

Fig. 8. Age histogram of the sample stars.

respectively (Fig. A.1). The iron content for the thin-disc members ranges from $[\mathrm{Fe} \mathrm{I} / \mathrm{H}] \approx-0.55$ to $0.35 \mathrm{dex}$, whereas the thickdisc members exhibit lower metallicity from $[\mathrm{Fe} \mathrm{I} / \mathrm{H}] \approx-0.8$ to -0.2 dex. The separation is most prominent in the case of magnesium. The sequences of element abundances in the two discs are distinguishable for $\mathrm{Si}, \mathrm{Ca}, \mathrm{Sc}$, and $\mathrm{Ti}$ as well. The trends of our results are in agreement with other studies (e.g. Adibekyan et al. 2012; Bensby et al. 2014; Mikolaitis et al. 2014). The two populations overlap more when looking at the calcium abundances which is in agreement with other studies (e.g. Bensby et al. 2005; Neves et al. 2009).

In Fig. A. 2 we follow variations of $\mathrm{Mg}, \mathrm{Si}, \mathrm{Ca}, \mathrm{Sc}$, and $\mathrm{Ti}$ with age. We observe positive slopes of $\left[\mathrm{El} / \mathrm{Fe}_{\mathrm{I}}\right]$ in relation to age for $\mathrm{Mg}$ and $\mathrm{Si}$ and almost no slopes for $\mathrm{Ca}, \mathrm{Sc}$, and Ti. A number of works that studied gradients of elemental abundances versus age is very small. However, we compared our values with the studies by Spina et al. (2016) and Nissen et al. (2017), who analysed the $[\mathrm{El} / \mathrm{Fe}]$ ratios for elements up to $\mathrm{Zn}$ as a function of stellar ages for solar twins whose age range is similar to ours. In Table 4 we provide slopes of linear fits $\Delta[\mathrm{El} / \mathrm{Fe}] / \Delta$ Age and compare them with the results by Spina et al. (2016) and Nissen et al. (2017). The gradients we obtained for $\mathrm{Mg}, \mathrm{Si}, \mathrm{Sc}$, and $\mathrm{Ti}$ are in general agreement with those in Spina et al. (2016) and Nissen et al. (2017). In the case of calcium, they provide a small negative slope, whereas we find a small positive slope. However, our slope values and those of Spina et al. (2016) and Nissen et al. (2017) for the Ca versus age relation are barely significant (less than $2 \sigma)$.

The GCE models for Sc and Ti completely fail to represent the data as it is shown in Figs. 9 and 10. Scandium is incorrectly modelled as underabundand by three out of the four models ( $\mathrm{K}_{\text {thin }}$ was not available for scandium), due to the deficiency of scandium in all sets of massive star yields of GCE studies (see also Kobayashi et al. 2006; Kobayashi \& Nakasato 2011; Nomoto et al. 2013). The PB model has the smallest deviation from our data (about $\approx 0.25 \mathrm{dex}$ ), whereas the other models predict very negative $[\mathrm{Sc} / \mathrm{Fe}]$ abundances for thin-disc $(\approx-0.5$ and less). The titanium model trends $[\mathrm{Ti} / \mathrm{Fe}]$ are also very negative. According to the work by Prantzos et al. (2018), a proper analysis of the physical conditions in which ${ }^{48} \mathrm{Cr}$ is produced should be more carefully investigated because the main isotope of $\mathrm{Ti}$ $\left.{ }^{48} \mathrm{Ti}\right)$ comes from the decay of ${ }^{48} \mathrm{Cr}$.

The computations for $\mathrm{Mg}, \mathrm{Si}$, and $\mathrm{Ca}$ are significantly more accurate; some models are at the level of the data (Figs. A.1 and A.2). Magnesium is predicted to be significantly less abundant by all models except $\mathrm{K}_{\text {thin }}$. Prantzos et al. (2018) admitted that isotopic abundances obtained for the time of the solar system formation are underproduced with the yields they adopted. The same is true for model R1, which uses the Woosley \& Weaver (1995) yields. Model R15 by Romano et al. (2010), which includes the yields from Kobayashi et al. (2006), can help reproduce the $\mathrm{Mg}$ evolution better, but still not well enough. On the other hand, the Kobayashi \& Nakasato (2011) $\mathrm{K}_{\text {thin }}$ model reproduces the abundances quite well in both $[\mathrm{Mg} / \mathrm{Fe} \mathrm{I}]$ versus $\mathrm{Fe}_{\mathrm{I}}$ and $\left[\mathrm{Mg} / \mathrm{Fe}_{\mathrm{I}}\right]$ versus age distributions. However, all the models that we plot can reproduce the $[\mathrm{Mg} / \mathrm{Fe} \mathrm{I}]$ trends (both in $\mathrm{Fe}_{\mathrm{I}}$ and age diagrams) quite well. Silicon versus $\mathrm{Fe}_{\mathrm{I}}$ is quite well reproduced by the PB and R1 models, whereas $\mathrm{R} 15$ and $\mathrm{K}_{\text {thin }}$ tend to overestimate the silicon-to-iron ratio; this ratio is reproduced very well versus age, except for the $\mathrm{K}_{\text {thin }}$ model. Calcium is reproduced better with the $\mathrm{PB}$ and $\mathrm{K}_{\text {thin }}$ models in both plots. All four models are predict an increase in $[\mathrm{Ca} / \mathrm{Fe} \mathrm{I}]$ towards older ages, which agrees with our thin-disc results. 


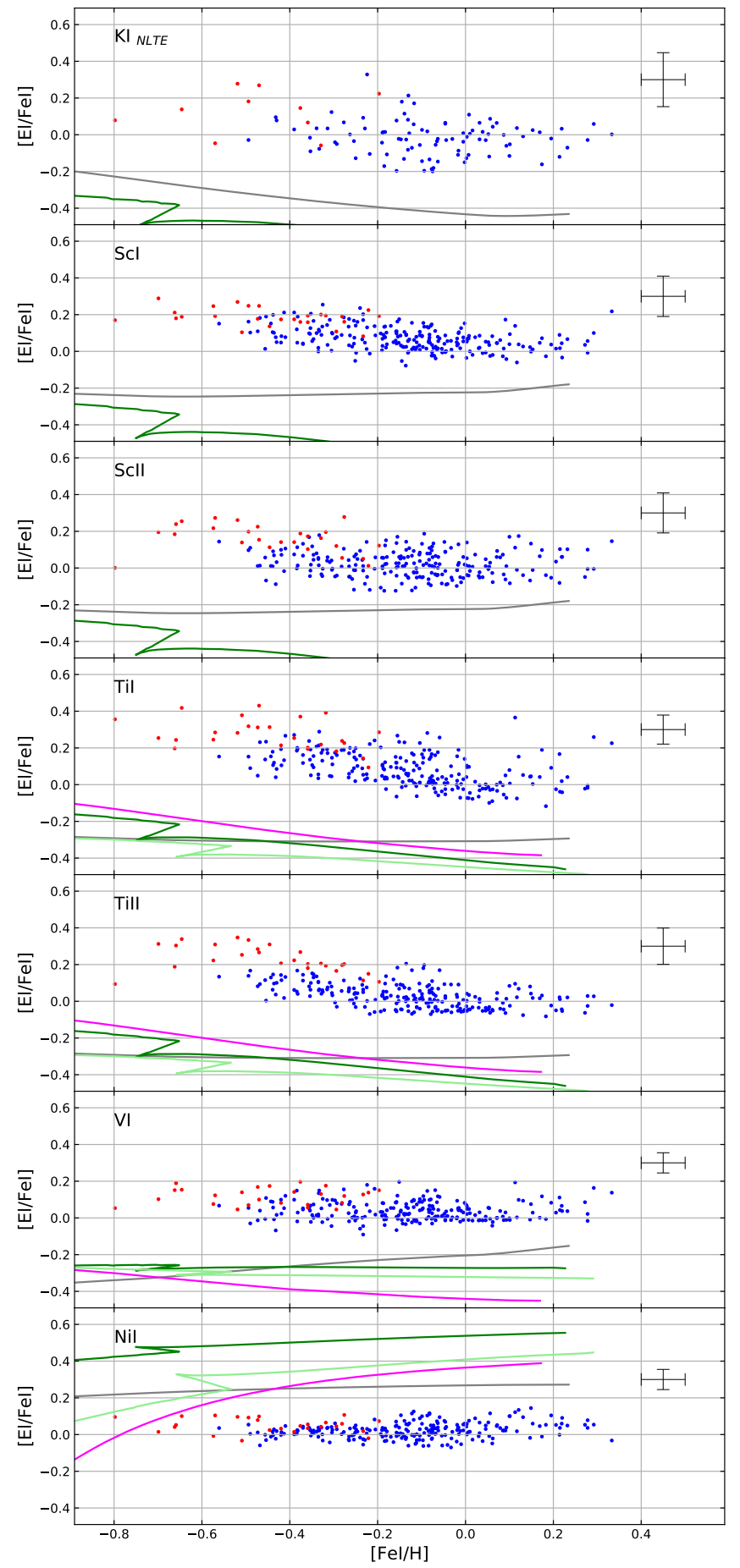

Fig. 9. Observed element-to-iron abundance ratios as a function of the $[\mathrm{Fe} \mathrm{I} / \mathrm{H}]$ abundance. Cases where all models fail to represent the data. Shown are the Romano et al. (2010) R1 model (dark green line) and R15 model (light green line), the Kobayashi \& Nakasato (2011) thin disc model (magenta line), and the Prantzos et al. (2018) baseline model (grey line).

\section{2. $\mathrm{Na}$ and $\mathrm{Al}$}

The sodium abundance seems to have a mild trend that decreases until $[\mathrm{Fe} \mathrm{I} / \mathrm{H}] \approx-0.2$, and then goes back up for the solar and metal-rich stars (Fig. A.1). We did not observe a significant distinction between thin- and thick-disc stars. As expected, the trend of $\left[\mathrm{Al} / \mathrm{I} / \mathrm{Fe}_{\mathrm{I}}\right]$ is similar to those found for the $\alpha$-elements.

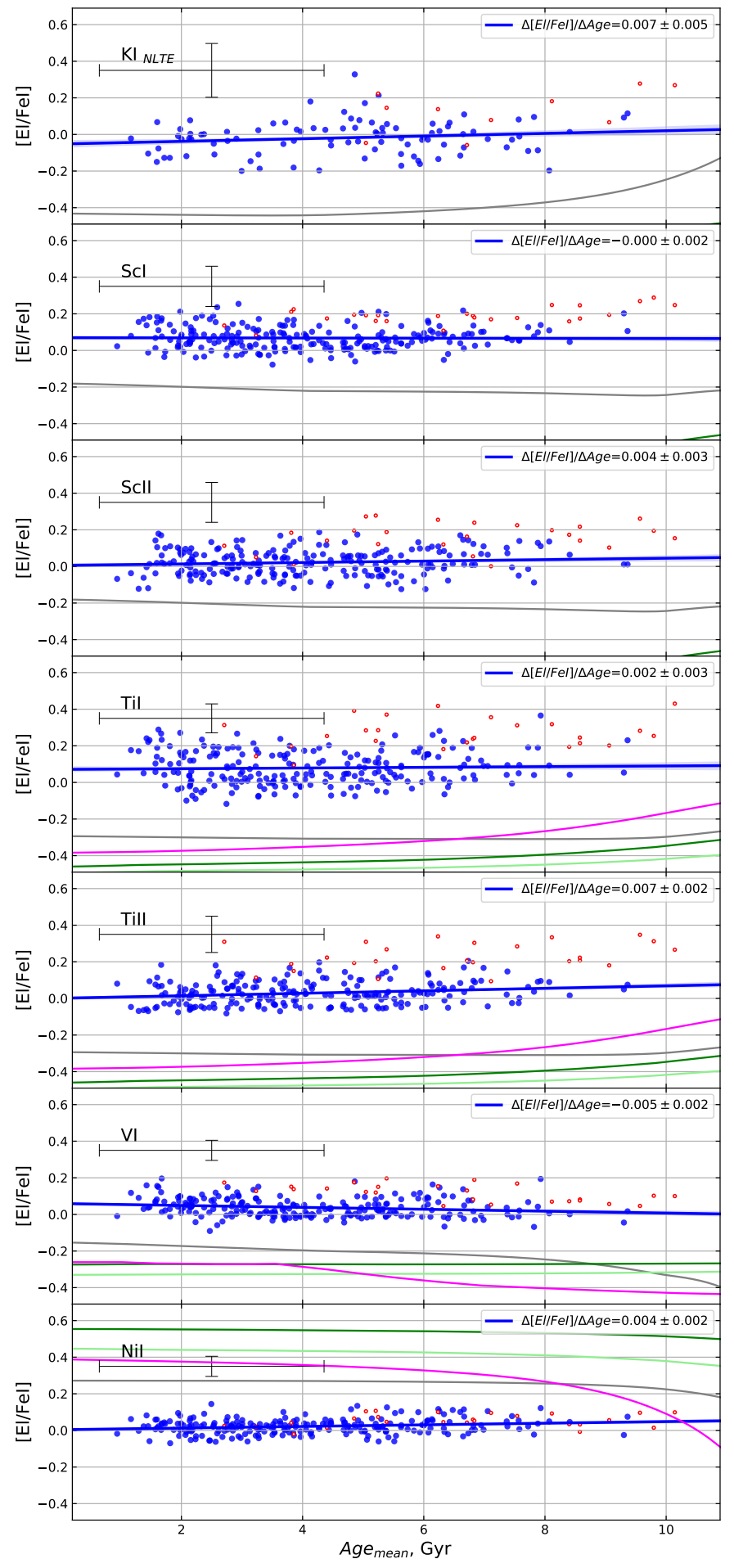

Fig. 10. Observed element-to-iron abundance ratios as a function of age for the cases where all models fail to represent the data. The blue line shows the $\Delta[\mathrm{El} / \mathrm{FeI}] / \Delta$ Age gradient. All other notations are as in Fig. 9.

The distributions are clearly distinct, with the thick-disc aluminium abundances generally being higher than the thin-disc values. There is a significant scatter in both thick- and thin-disc sequences. In general, the behaviour of $\mathrm{Na}$ and $\mathrm{Al}$ are similar to those obtained by Neves et al. (2009), Adibekyan et al. (2012), and Bensby et al. (2014).

For sodium-to-iron abundance ratios we obtain a slightly negative slope in comparison with ages (Fig. A.2 and Table 4), 


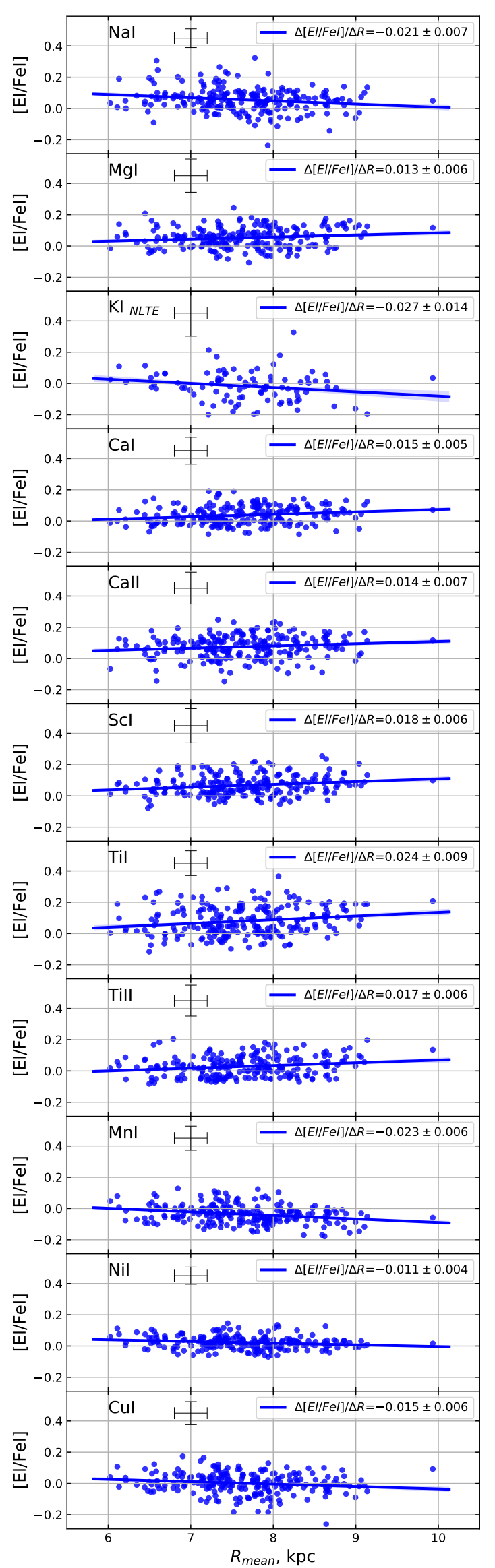

Fig. 11. Observed element-to-iron abundance ratios as a function of $R_{\text {mean }}($ significance $>2 \sigma)$.

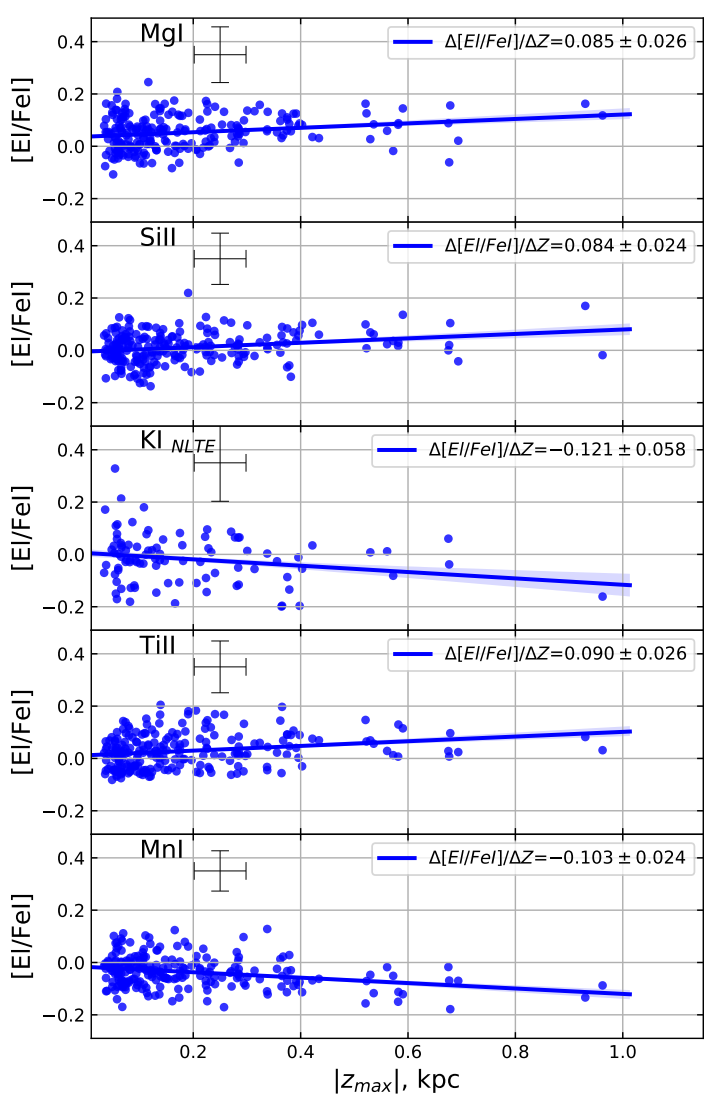

Fig. 12. Observed element-to-iron abundance ratios as a function of $z_{\max }$ (significance $>2 \sigma$ ).

whereas Spina et al. (2016) and Nissen et al. (2017) showed a positive slope. For aluminium, in agreement with Spina et al. (2016) and Nissen et al. (2017), we find clear positive slopes that are similar to those for magnesium.

Sodium is predicted to be underabundant by the R1 and PB models, whereas R15 (which includes the Kobayashi et al. 2006 yields) agrees better with the data. Interestingly, the $\mathrm{K}_{\mathrm{thin}}$ model is significantly overabundant. The PB model shows a slightly negative abundance trend in relation to age, as can be seen in our data as well. Aluminium is similarly well predicted by the $\mathrm{R} 1$ and R15 models, whereas the PB model is underabundant by $\approx 0.2$ dex and the $K_{\text {thin }}$ is overabundant in comparison with our data. However, while being overabundant, only $\mathrm{K}_{\text {thin }}$ shows a similar positive trend of $\mathrm{Al}$ abundance versus age as determined in this work and in Spina et al. (2016).

\section{3. $S$ and $K$}

In $\left[\mathrm{S}_{\mathrm{I}} / \mathrm{Fe}_{\mathrm{I}}\right]$ versus $\left[\mathrm{Fe}_{\mathrm{I}} / \mathrm{H}\right]$ space we observe that sulphur abundances behave similarly to $\alpha$-elements. This trend is also seen in Mishenina et al. (2015). Potassium abundances show larger scatter with some minor decrease towards larger $[\mathrm{Fe} \mathrm{I} / \mathrm{H}]$. Generally, the $\left[\mathrm{K}_{\left.\mathrm{I}_{\text {NLTE }} / \mathrm{Fe} \mathrm{I}\right]}\right.$ versus $\left.[\mathrm{Fe} \mathrm{I}] / \mathrm{H}\right]$ behaviour is similar to those reported by Takeda et al. (2002) and Zhao et al. (2016).

The slope of sulphur distribution versus age is mildly positive and agrees well with the values provided by both Spina


tion versus age is slightly positive, but of low significance. Spina et al. (2016) did not provide any slopes for potassium, which seems to have no definite dependence on age, probably because its abundance determinations are based on the large saturated 
Table 4. Best linear fits to the $[\mathrm{El} / \mathrm{Fe}]-$ age distributions in dex $\mathrm{Gyr}^{-1}$.

\begin{tabular}{|c|c|c|c|}
\hline Elements & $\begin{array}{l}\text { This work } \\
\text { thin-disc }\end{array}$ & $\begin{array}{c}\text { Spina et al. (2016) } \\
\text { solar twins }\end{array}$ & $\begin{array}{c}\text { Nissen et al. (2017) } \\
\text { solar twins }\end{array}$ \\
\hline$\left[\mathrm{Na} / / \mathrm{Fe}_{\mathrm{I}}\right]$ & $-0.006 \pm 0.003$ & $0.0150 \pm 0.0030$ & $0.0089 \pm 0.0025$ \\
\hline$\left[\mathrm{Mg}_{\mathrm{I}} / \mathrm{Fe}_{\mathrm{I}}\right]$ & $0.016 \pm 0.002$ & $0.0100 \pm 0.0009$ & $0.0095 \pm 0.0007$ \\
\hline$\left[\mathrm{Al} / \mathrm{I} / \mathrm{Fe}_{\mathrm{I}}\right]$ & $0.019 \pm 0.003$ & $0.0147 \pm 0.0015$ & $0.0148 \pm 0.0011$ \\
\hline$[\mathrm{Si} / \mathrm{Fe} \mathrm{I}]$ & $0.009 \pm 0.002$ & $0.0051 \pm 0.0008$ & $0.0037 \pm 0.0007$ \\
\hline [Si II/Fe I] & $0.011 \pm 0.002$ & & \\
\hline$\left[\mathrm{S}_{\mathrm{I}} / \mathrm{Fe}_{\mathrm{I}}\right]$ & $0.004 \pm 0.003$ & $0.0046 \pm 0.0017$ & $0.0046 \pm 0.0010$ \\
\hline$\left[\mathrm{KI}_{\mathrm{I}} / \mathrm{Fe}_{\mathrm{I}}\right]$ & $-0.027 \pm 0.006$ & & \\
\hline$\left[\mathrm{Ca} I / \mathrm{Fe}_{\mathrm{I}}\right]$ & $0.001 \pm 0.002$ & $-0.0014 \pm 0.0008$ & $-0.0008 \pm 0.0003$ \\
\hline$[\mathrm{Ca}$ II $/ \mathrm{Fe}$ I] & $0.005 \pm 0.003$ & & \\
\hline$\left[\mathrm{Sc}_{\mathrm{I}} / \mathrm{Fe}_{\mathrm{I}}\right]$ & $0.000 \pm 0.002$ & $0.0071 \pm 0.0018$ & \\
\hline$[\mathrm{Sc} \mathrm{II} / \mathrm{Fe} \mathrm{I}]$ & $0.004 \pm 0.003$ & & \\
\hline [Ti I/Fe I] & $0.002 \pm 0.003$ & $0.0061 \pm 0.0011$ & $0.0054 \pm 0.0006$ \\
\hline$[\mathrm{Ti} \mathrm{II} / \mathrm{Fe} \mathrm{I}]$ & $0.007 \pm 0.002$ & & \\
\hline$\left[\mathrm{V}_{\mathrm{I}} / \mathrm{Fe}_{\mathrm{I}}\right]$ & $-0.005 \pm 0.002$ & $-0.0034 \pm 0.0012$ & \\
\hline$[\mathrm{Cr} \mathrm{I} / \mathrm{Fe} \mathrm{I}]$ & $0.001 \pm 0.002$ & $-0.0024 \pm 0.0005$ & $-0.0013 \pm 0.0005$ \\
\hline$[\mathrm{Cr}$ II/Fe I $]$ & $-0.003 \pm 0.002$ & & \\
\hline$[\mathrm{Mn} \mathrm{I} / \mathrm{Fe} \mathrm{I}]$ & $-0.010 \pm 0.002$ & $0.0075 \pm 0.0019$ & \\
\hline$\left[\mathrm{Co} / / \mathrm{Fe}_{\mathrm{I}}\right]$ & $0.005 \pm 0.003$ & $0.0120 \pm 0.0030$ & \\
\hline$[\mathrm{Ni}$ I/Fe I] & $0.004 \pm 0.002$ & $0.0076 \pm 0.0017$ & $0.0058 \pm 0.0012$ \\
\hline$\left[\mathrm{Cu} / / \mathrm{Fe}_{\mathrm{I}}\right]$ & $-0.004 \pm 0.002$ & $0.0200 \pm 0.0030$ & \\
\hline$\left[\mathrm{Zn} \mathrm{I} / \mathrm{Fe}_{\mathrm{I}}\right]$ & $-0.001 \pm 0.003$ & $0.0122 \pm 0.0014$ & $0.0112 \pm 0.0009$ \\
\hline
\end{tabular}

line at $7698.97 \AA$ (e.g. $\approx 154 \mathrm{~m} \AA$ for the Sun, Moore et al. 1966), which is more suited for the analysis by spectral synthesis.

Potassium abundances (Figs. 9 and 10) are underestimated by all four models (PB, R1, R15, and $\mathrm{K}_{\text {thin }}$ ); the differences are $\approx 0.25$ dex. The two models that are based on Kobayashi et al. (2006) and Kobayashi \& Nakasato (2011) (i.e. $K_{\text {thin }}$, R15) yields underestimated potassium abundances (by $>0.65 \mathrm{dex}$ ), and they are even out of range in our plots. The $\mathrm{PB}$ and $\mathrm{R} 1$ models predict a mild positive slope of $\left[\mathrm{K}_{\mathrm{I}_{\mathrm{NLTE}}} / \mathrm{Fe}_{\mathrm{I}}\right]$ versus age, which is also seen in our data.

Sulphur behaviour in the thin-disc is better predicted by the PB and $K_{\text {thin }}$ models (see Figs. A.1 and A.2). The $K_{\text {thin }}$ model most closely reproduces the results. The mild positive slope of abundance ratios versus age is seen by all four models; however, $\mathrm{R} 1$ and $\mathrm{R} 15$ are underabundant by $\approx 0.2$ dex.

\subsection{V, Cr, Co, Ni, Mn}

The V- and Co-to-Fe ratios in the thin- and thick-disc stars resemble those of $\alpha$-elements, whereas $\mathrm{Cr}$ and $\mathrm{Ni}$ abundances are well confined around the $\mathrm{Fe}_{\mathrm{I}}$ value. The manganese-to-iron ratio trends are opposite to those of $\alpha$-elements for the reasons mentioned above. In general, the LTE abundance trends of these iron-peak elements are similar to those obtained by Neves et al. (2009), Adibekyan et al. (2012), Bensby et al. (2014), and Battistini \& Bensby (2015).

The slopes of [El/Fe I] versus the age distributions of $\mathrm{Cr}, \mathrm{V}$, $\mathrm{Co}$, and $\mathrm{Ni}$ are not very steep and in general agreement with those from studies by Spina et al. (2016) and Nissen et al. (2017). The slope of manganese abundance versus age determined in this work is actually opposite to that of solar twins by Spina et al. (2016), which is expected as a majority of the older stars are found at the metal-poor end of the thin-disc where the lowest manganese-to-iron ratios are.

The elements $\mathrm{V}$ and $\mathrm{Ni}$ are other examples where all models fail to represent the data (Figs. 9 and 10). Vanadium models underestimate the observed abundances by at least $\approx 0.2$ dex. The slight negative slope is predicted by the PB and $\mathrm{K}_{\text {thin }}$ mod- els, which is in agreement with our study and the results from Spina et al. (2016). Nickel is overestimated by all the models we use, and the disagreement between the results and theoretical calculations is very large, reaching more than 0.4 dex for the Romano et al. (2010) models and $\approx 0.27$ dex for the Prantzos et al. (2018) PB model (with $\mathrm{K}_{\text {thin }}$ being in between). All models predict a slightly negative slope in the $[\mathrm{Ni} / \mathrm{Fe}$ I] versus age relation, which disagree with our results and with those by other authors (Spina et al. 2016; Nissen et al. 2017).

Chromium is modelled quite well by the PB, R15, and $\mathrm{K}_{\text {thin }}$ models; however, R1 shows a minor overabundance. The manganese abundance in the thin-disc is better reproduced by the PB, R15, and $\mathrm{K}_{\text {thin }}$ models. All models predict the negative slope of $[\mathrm{Mn} \mathrm{I} / \mathrm{Fe} \mathrm{I}]$ versus age relation. The results of cobalt favour the R1 model, whereas the other three seem to underestimate its abundance. The slope of $[\mathrm{Mn} \mathrm{I} / \mathrm{Fe} \mathrm{I}]$ versus age is better recreated by the R1 model. The slope from the PB seems to be negative, while our data and that by Spina et al. (2016) show some positive tendency.

\section{5. $\mathrm{Cu}, \mathrm{Zn}$}

In Fig. A.1 we see that the Galactic components have distinct behaviours in the $\left[\mathrm{Cu} / / \mathrm{Fe}_{\mathrm{I}}\right]$ versus $\left[\mathrm{Fe}_{\mathrm{I}} / \mathrm{H}\right]$ plane. The thickdisc stars look slightly more enriched in $\mathrm{Cu}$ than the thin-disc stars and the thin-disc exhibits a shallow increase in $[\mathrm{Cu} \mathrm{I} / \mathrm{Fe} \mathrm{I}]$ at super-solar metallicities. Our copper trends are similar to those from other studies (Bensby et al. 2014; Mikolaitis et al. 2017; Delgado Mena et al. 2017), whereas zinc has an $\alpha$-like behaviour that was already reported in some previous studies (Bisterzo et al. 2006; Saito et al. 2009; Mishenina et al. 2011; Bensby et al. 2014; Mikolaitis et al. 2017; Delgado Mena et al. 2017). As is seen in Fig. A.2 and Table 4, the copper and zinc distributions display almost no trends with the age, which disagree with the studies of solar twins by Spina et al. (2016) and Nissen et al. (2017), who report clear positive slopes.

Copper is better reproduced by the $\mathrm{R} 1$ model, whereas $\mathrm{R} 15$ and $\mathrm{PB}$ underestimate $[\mathrm{Cu} \mathrm{I} / \mathrm{Fe} \mathrm{I}]$ ratios and $\mathrm{K}_{\text {thin }}$ does the opposite and predicts values that are a bit higher (Figs. A.1 and A.2). All models predict negative slopes in relation to age and that is in broad agreement with our data; however, solar twins behave differently (Spina et al. 2016). Zinc favours the R15 model in element abundance versus metallicity and versus age relations. R1 predicts the unrealistic underabundance towards lower metallicities and the PB model generally shows a strong underabundance by $\approx 0.25$ dex, while $\mathrm{K}_{\text {thin }}$ is slightly overabundant at higher metallicities.

\section{Radial and vertical abundance gradients in the thin-disc}

Figures 11 and 12 display the $[\mathrm{El} / \mathrm{Fe} \mathrm{I}]$ significant gradients $(>2 \sigma)$ in relation to the mean Galactocentric distance $\left(R_{\text {mean }}\right)$ and the maximum height above the Galactic plane $\left(\left|z_{\max }\right|\right)$ in the thin-disc sample, while all numerical values of the gradients are presented in Table 5 . We find positive slopes $(>2 \sigma)$ for most of the $\alpha$-elements: $\mathrm{Mg}$ I, Ca I, Ca II,Sc I, Ti I, Ti II. On the other hand, Na I, Mn I, Ni I, and $\mathrm{Cu}$ I show a significant decrease in abundance with increasing Galactocentric distances.

When looking at the $\Delta[\mathrm{X} / \mathrm{Fe}] / \Delta z_{\max }$ relations, the situation is similar. The $\alpha$-elements generally have positive slopes with Mg I, Si II, Sc II, and Ti II being significant by more than $>2 \sigma$, whereas only $\mathrm{Mn}$ I shows a significant negative trend $(4.2 \sigma)$. The 
Table 5. Gradients in relation to the mean Galactocentric distance $\left(R_{\text {mean }}\right)$ and the maximum height above the Galactic plane $\left(\left|z_{\max }\right|\right)$ in the thin-disc sample.

\begin{tabular}{lcc}
\hline \hline Element & $\begin{array}{c}\Delta[\mathrm{X} / \mathrm{Fe}] / \Delta R_{\text {mean }} \\
\mathrm{dex} \mathrm{kpc}^{-1}\end{array}$ & $\begin{array}{c}\Delta[\mathrm{X} / \mathrm{Fe}] / \Delta z_{\max } \\
\mathrm{dex} \mathrm{kpc}^{-1}\end{array}$ \\
\hline $\mathrm{Na}_{\text {I }}$ & $-0.021 \pm 0.007$ & $-0.024 \pm 0.032$ \\
$\mathrm{Mg}_{\text {I }}$ & $0.013 \pm 0.006$ & $0.085 \pm 0.026$ \\
$\mathrm{Al}$ I & $-0.007 \pm 0.008$ & $0.061 \pm 0.034$ \\
$\mathrm{Si}$ I & $0.002 \pm 0.005$ & $0.040 \pm 0.021$ \\
$\mathrm{Si}_{\text {II }}$ & $0.009 \pm 0.006$ & $0.084 \pm 0.024$ \\
$\mathrm{~S}_{\text {I }}$ & $-0.003 \pm 0.008$ & $0.001 \pm 0.035$ \\
$\mathrm{~K}_{\text {INLTE }}$ & $-0.027 \pm 0.014$ & $-0.121 \pm 0.058$ \\
$\mathrm{Ca}_{\text {I }}$ & $0.015 \pm 0.005$ & $-0.002 \pm 0.024$ \\
$\mathrm{Ca}_{\text {II }}$ & $0.014 \pm 0.007$ & $0.039 \pm 0.030$ \\
$\mathrm{Sc}_{\text {I }}$ & $0.018 \pm 0.006$ & $0.012 \pm 0.026$ \\
$\mathrm{Sc}_{\text {II }}$ & $0.006 \pm 0.007$ & $0.110 \pm 0.028$ \\
$\mathrm{Ti}_{\text {I }}$ & $0.024 \pm 0.009$ & $0.014 \pm 0.038$ \\
$\mathrm{Ti}_{\text {II }}$ & $0.017 \pm 0.006$ & $0.090 \pm 0.026$ \\
$\mathrm{~V}_{\text {I }}$ & $0.002 \pm 0.005$ & $-0.017 \pm 0.022$ \\
$\mathrm{Cr}_{\text {I }}$ & $0.007 \pm 0.004$ & $-0.008 \pm 0.018$ \\
$\mathrm{Cr}_{\text {II }}$ & $0.008 \pm 0.006$ & $0.009 \pm 0.026$ \\
$\mathrm{Mn}_{\text {I }}$ & $-0.023 \pm 0.006$ & $-0.103 \pm 0.024$ \\
$\mathrm{Co}_{\text {I }}$ & $0.000 \pm 0.007$ & $0.060 \pm 0.029$ \\
$\mathrm{Ni}_{\text {I }}$ & $-0.011 \pm 0.004$ & $0.013 \pm 0.017$ \\
$\mathrm{Cu}_{\text {I }}$ & $-0.015 \pm 0.006$ & $0.030 \pm 0.028$ \\
$\mathrm{Zn}_{\text {I }}$ & $0.004 \pm 0.007$ & $0.039 \pm 0.031$ \\
\hline
\end{tabular}

case of $\mathrm{K}_{\mathrm{I}_{\mathrm{NLTE}}}$ is interesting: it shows negative slopes in relation to $R_{\text {mean }}$ and to $\left|z_{\max }\right|$, which are significant by $\approx 2 \sigma$.

There are no published data on $[\mathrm{El} / \mathrm{Fe}]$ gradients in relation to $R_{\text {mean }}$ or $\left|z_{\max }\right|$. Typically, elemental abundance gradients are studied by large spectroscopic surveys that observe more distant stars, and they usually study gradients according to the current $R_{\mathrm{GC}}$ and $|z|$ or the guiding radii $\left(R_{\mathrm{g}}\right)$. However, even large spectroscopic surveys have published very few elemental radial or vertical gradients for field stars separated into thin- or thick-disc components.

The data of RAVE, APOGEE, and GES are quite different from our (different target selection, different distance estimation methods etc.); however, the gradients determined by Boeche et al. (2013), Bergemann et al. (2014), and Mikolaitis et al. (2014) qualitatively agree with ours (Table 6). The thin-disc abundance gradients can be compared with the studies based on Cepheid variables as well. For example, slightly positive gradients of individual $\alpha$-elements seem to agree with the work by Genovali et al. (2015). Since the Cepheids are young metal-rich stars, they show the present-day gradients (Cescutti et al. 2007), thus we can only search for a broad agreement to our results.

Unfortunately, there are few elemental abundance vertical gradients reported in the literature, and they are mostly based on $\alpha$-elements. The GES GIRAFFE-based study by Mikolaitis et al. (2014) and Li et al. (2018) from the APOGEE survey obtained generally positive gradients in the thin-disc sample, while the GALAH survey (Duong et al. 2018) determined only a shallow positive gradient of $\Delta[\alpha / \mathrm{Fe}] / \Delta z$.

\section{Summary}

This paper is the second release of spectroscopic data from the Spectroscopic and Photometric Survey of the Northern Sky (SPFOT). The survey aims to provide a detailed chemical composition from high-resolution spectra and photometric variabil- ity data for bright stars in the northern sky using telescopes at the Molètai Astronomical Observatory, Vilnius University.

We observed high-resolution spectra for all 192 photometrically selected $6500 \mathrm{~K}$ and cooler dwarfs in the sky area with radius of $20^{\circ}$ centred on the preliminary ESA-PLATO NPF field and derived the main atmospheric parameters for 109 stars. We co-added this sample with 140 stars from the STEP 2 field investigated in Paper I and computed kinematic parameters, ages, and abundances of 21 chemical species for 249 FGK stars in total. These regions of the sky are becoming very important as they will soon be intensively observed by the NASA TESS mission (Ricker et al. 2015; Sullivan et al. 2015).

We turned our attention to bright stars since only up to $30 \%$ of the stars that are brighter than 8 mag have some previous spectroscopic observations. Apart from faint and distant stars, which are more often observed by large spectroscopic surveys, it is important to pay attention to the bright nearby stars as well, especially for the objects in the "hot" regions like the TESS continuous viewing zones (Sharma et al. 2018), and possible PLATO fields (Miglio et al. 2017).

With this study we also examined whether bright solar vicinity stars can provide important information about the Galactic chemical evolution. We used the $[\mathrm{Mg} / \mathrm{Fe}]$ ratio to divide the sample stars to the thin-disc (223 stars) and thick-disc (26 stars). We then explored the behaviour of 21 chemical species in $[\mathrm{El} / \mathrm{Fe} \mathrm{I}]$ versus $[\mathrm{Fe} \mathrm{I} / \mathrm{H}]$ and $\left[\mathrm{El} / \mathrm{Fe}_{\mathrm{I}}\right]$ versus age planes. We restricted our conclusions to the thin-disc stars since the sample of thick-disc stars still should be increased. Thus, for the thin-disc stars we found that $\alpha$-elements and aluminium abundances have positive trends with age, while the trend of the Mn abundance is obviously negative.

In order to better understand the chemical evolution of the solar neighbourhood, we compared our observational results to the recent theoretical models by Romano et al. (2010), Kobayashi \& Nakasato (2011), and Prantzos et al. (2018) that provide predictions for a variety of chemical elements (up to $Z=30$ ) not only in the $\left[\mathrm{El} / \mathrm{Fe}_{\mathrm{I}}\right]$ versus $[\mathrm{Fe} \mathrm{I} / \mathrm{H}]$ plane, but also in the $\left[\mathrm{El} / \mathrm{Fe}_{\mathrm{I}}\right]$ versus age plane. The comparison between the observed data and the theoretical models led to the conclusion that it is still difficult for scientists to model the nucleosynthesis channels for potasium, scandium, titanium, vanadium, and nickel. Even if adjustments in some metallicity domains are still required, other elements are modelled rather well.

In our study, we attempted to estimate elemental abundance ([El/Fe $\mathrm{I}]$ ) gradients versus the mean Galactocentric distance and height above the Galactic plane. Our stars are in close proximity to the Sun $\left(7.9<R_{\mathrm{GC}}<8.2 \mathrm{kpc}, 0.02<|z|<0.14 \mathrm{kpc}\right)$; however, their orbital parameters revealed quite wide ranges of their mean Galactocentric distances and maximum heights above the Galactic plane $\left(5.91<R_{\text {mean }}<10.51 \mathrm{kpc}, 0.03<\left|z_{\max }\right|<\right.$ $1.29 \mathrm{kpc})$. We prove that using $R_{\text {mean }}$ and $\left|z_{\max }\right|$ is very helpful while estimating the gradients of element abundance ratios in the Galactic disc. We found that the $\alpha$-element and zinc abundances have slightly positive or flat radial and vertical gradients, while gradients for the odd- $Z$ element $\mathrm{Na}, \mathrm{K}, \mathrm{V}$, and $\mathrm{Mn}$ abundances are negative.

The study of vertical and radial abundance gradients in the Galactic discs, as well as the age dependence of element abundances can provide strong evidence of the mechanisms of Galaxy formation and should be continued.

Acknowledgements. This research has made use of the SIMBAD database and NASA Astrophysics Data System (operated at CDS, Strasbourg, France). This work has made use of data from the European Space Agency (ESA) mission Gaia 
Table 6. Radial and vertical [El/Fe] gradients in the Galactic thin-disc from literature.

\begin{tabular}{|c|c|c|c|c|}
\hline Elements & Value $\left(\mathrm{dex} \mathrm{kpc} \mathrm{kp}^{-1}\right)$ & Source & Definition of thin-disc & Comments \\
\hline \multicolumn{5}{|l|}{ Galactocentric } \\
\hline$\Delta[\alpha / \mathrm{Fe}] / \Delta R_{\mathrm{g}}$ & $-0.004 \pm 0.001$ & Boeche et al. (2013) & $\left|Z_{\max }\right|<0.04 \mathrm{kpc}$ & RAVE sample \\
\hline$\Delta[\alpha / \mathrm{Fe}] / \Delta R_{\mathrm{g}}$ & $0.010 \pm 0.002$ & Boeche et al. (2013) & $\left|Z_{\max }\right|<0.04 \mathrm{kpc}$ & GCS sample \\
\hline$\Delta[\alpha / \mathrm{Fe}] / \Delta R_{\mathrm{GC}}$ & $-0.005 \pm 0.002$ & Anders et al. (2014) & $0.0<|z|<0.4 \mathrm{kpc}$ & APOGEE gold sample \\
\hline$\Delta[\mathrm{Mg} \mathrm{I} / \mathrm{Fe}] / \Delta R_{\mathrm{GC}}$ & $0.021 \pm 0.016$ & Bergemann et al. (2014) & $0.0<|z|<0.3 \mathrm{kpc}$ & GES UVES \\
\hline$\Delta\left[\mathrm{Mg}_{\mathrm{I}} / \mathrm{Fe}\right] / \Delta R_{\mathrm{GC}}$ & $0.009 \pm 0.003$ & Mikolaitis et al. (2014) & Chemical & GES GIRAFFE \\
\hline$\Delta[\mathrm{Si} \mathrm{I} / \mathrm{Fe}] / \Delta R_{\mathrm{GC}}$ & $0.021 \pm 0.004$ & Mikolaitis et al. (2014) & Chemical & GES GIRAFFE \\
\hline$\Delta[\mathrm{Ca} \mathrm{II} / \mathrm{Fe}] / \Delta R_{\mathrm{GC}}$ & $0.017 \pm 0.004$ & Mikolaitis et al. (2014) & Chemical & GES GIRAFFE \\
\hline$\Delta[\mathrm{Na} / \mathrm{Fe}] / \Delta R_{\mathrm{GC}}$ & $0.007 \pm 0.002$ & Genovali et al. (2015) & & Cepheids \\
\hline$\Delta[\mathrm{Na} / \mathrm{Fe}] / \Delta R_{\mathrm{GC}}$ & $0.001 \pm 0.002$ & Genovali et al. (2015) & & Cepheids \\
\hline$\Delta[\mathrm{Mg} / \mathrm{Fe}] / \Delta R_{\mathrm{GC}}$ & $0.013 \pm 0.003$ & Genovali et al. (2015) & & Cepheids \\
\hline$\Delta[\mathrm{Si} / \mathrm{Fe}] / \Delta R_{\mathrm{GC}}$ & $0.007 \pm 0.002$ & Genovali et al. (2015) & & Cepheids \\
\hline$\Delta[\mathrm{Ca} / \mathrm{Fe}] / \Delta R_{\mathrm{GC}}$ & $0.018 \pm 0.002$ & Genovali et al. (2015) & & Cepheids \\
\hline \multicolumn{5}{|l|}{ Vertical } \\
\hline$\Delta[\mathrm{Mg} \mathrm{I} / \mathrm{Fe}] \Delta z$ & $0.041 \pm 0.004$ & Mikolaitis et al. (2014) & Chemical & GES GIRAFFE \\
\hline$\Delta[\mathrm{Al} \mathrm{I} / \mathrm{Fe}] \Delta z$ & $0.051 \pm 0.004$ & Mikolaitis et al. (2014) & Chemical & GES GIRAFFE \\
\hline$\Delta[\mathrm{Si}$ I $/ \mathrm{Fe}] \Delta z$ & $0.006 \pm 0.008$ & Mikolaitis et al. (2014) & Chemical & GES GIRAFFE \\
\hline$\Delta[\mathrm{Ca} \mathrm{II} / \mathrm{Fe}] \Delta z$ & $0.034 \pm 0.010$ & Mikolaitis et al. (2014) & Chemical & GES GIRAFFE \\
\hline$\Delta[\alpha / \mathrm{Fe}] / \Delta z$ & $0.008 \pm 0.002$ & Duong et al. (2018) & Chemical and kinematical & GALAH survey \\
\hline$\Delta[\alpha / \mathrm{Fe}] / \Delta z$ & $0.022 \pm 0.001$ & Li et al. (2018) & Kinematical & GES GIRAFFE \\
\hline
\end{tabular}

(https://www.cosmos.esa.int/gaia), processed by the Gaia Data Processing and Analysis Consortium (DPAC, https://www.cosmos.esa.int/ web/gaia/dpac/consortium). Funding for the DPAC has been provided by national institutions, in particular the institutions participating in the Gaia Multilateral Agreement. We are especially grateful to T. Masseron and B. Plez for providing us with molecular data. We appreciate that D. Romano, N. Prantzos, C. I. Johnson, and C. Kobayashi kindly shared their model data. This research was funded by the grant from the Research Council of Lithuania (LAT-08/2016).

\section{References}

Adibekyan, V. Z., Sousa, S. G., Santos, N. C., et al. 2012, A\&A, 545, A32 Alexeeva, S., Ryabchikova, T., Mashonkina, L., \& Hu, S. 2018, ApJ, 866, 153 Alvarez, R., \& Plez, B. 1998, A\&A, 330, 1109

Anders, F., Chiappini, C., Santiago, B. X., et al. 2014, A\&A, 564, A115 Andrievsky, S. M., Spite, M., Korotin, S. A., et al. 2008, A\&A, 481, 481 Battistini, C., \& Bensby, T. 2015, A\&A, 577, A9

Baumueller, D., \& Gehren, T. 1997, A\&A, 325, 1088

Bensby, T., Feltzing, S., \& Lundström, I. 2003, A\&A, 410, 527

Bensby, T., Feltzing, S., Lundström, I., \& Ilyin, I. 2005, A\&A, 433, 185

Bensby, T., Feltzing, S., \& Oey, M. S. 2014, A\&A, 562, A71

Bergemann, M. 2011, MNRAS, 413, 2184

Bergemann, M., \& Gehren, T. 2008, A\&A, 492, 823

Bergemann, M., Kudritzki, R.-P., Würl, M., et al. 2013, ApJ, 764, 115

Bergemann, M., Ruchti, G. R., Serenelli, A., et al. 2014, A\&A, 565, A89

Bergström, H., Peng, W. X., \& Persson, A. 1989, Z. Phys. D At. Mol. Clusters, 13,203

Bisterzo, S., Gallino, R., \& Pignatari, M. 2006, in Cu and Zn in Thick-Disk and Thin-Disk Stars, eds. S. Randich, \& L. Pasquini, 39

Blackwell-Whitehead, R. J., Pickering, J. C., Pearse, O., \& Nave, G. 2005, ApJS, 157,402

Boeche, C., Siebert, A., Piffl, T., et al. 2013, A\&A, 559, A59

Bovy, J. 2015, ApJS, 216, 29

Bovy, J., Allende Prieto, C., Beers, T. C., et al. 2012, ApJ, 759, 131

Bressan, A., Marigo, P., Girardi, L., et al. 2012, MNRAS, 427, 127

Brodzinski, T., Kronfeldt, H.-D., Kropp, J.-R., \& Winkler, R. 1987, Z. Phys. D At. Mol. Clusters, 7, 161

Brooke, J. S. A., Bernath, P. F., Schmidt, T. W., \& Bacskay, G. B. 2013, J. Quant. Spectr. Rad. Transf., 124, 11

Casagrande, L., Schönrich, R., Asplund, M., et al. 2011, A\&A, 530, A138 Cescutti, G., Matteucci, F., François, P., \& Chiappini, C. 2007, A\&A, 462, 943 Chen, Y. Q., Nissen, P. E., \& Zhao, G. 2004, A\&A, 425, 697

Davis, S. J., Wright, J. J., \& Balling, L. C. 1971, Phys. Rev. A, 3, 1220

Delgado Mena, E., Tsantaki, M., Adibekyan, V. Z., et al. 2017, A\&A, 606, A94
Dembczyński, J., Ertmer, W., Johann, U., Penselin, S., \& Stinner, P. 1979, Z. Phys. A Hadrons Nucl., 291, 207

Dulick, M., Bauschlicher, Jr., C. W., Burrows, A., et al. 2003, ApJ, 594, 651

Duong, L., Freeman, K. C., Asplund, M., et al. 2018, MNRAS, 476, 5216

Ekström, S., Meynet, G., Chiappini, C., Hirschi, R., \& Maeder, A. 2008, A\&A, 489, 685

Feuillet, D. K., Bovy, J., Holtzman, J., et al. 2018, MNRAS, 477, 2326

Fischer, W., Hühnermann, H., \& Kollath, K.-J. 1967, Z. Phys., 200, 158

Frasca, A., Guillout, P., Klutsch, A., et al. 2018, A\&A, 612, A96

Fuhrmann, K. 1998, A\&A, 338, 161

Fuhrmann, K. 2004, Astron. Nachr., 325, 3

Gaia Collaboration (Prusti, T., et al.) 2016, A\&A, 595, A1

Gaia Collaboration (Brown, A. G. A., et al.) 2018, A\&A, 616, A1

Gehren, T., Liang, Y. C., Shi, J. R., Zhang, H. W., \& Zhao, G. 2004, A\&A, 413, 1045

Gehren, T., Shi, J. R., Zhang, H. W., Zhao, G., \& Korn, A. J. 2006, A\&A, 451, 1065

Genovali, K., Lemasle, B., da Silva, R., et al. 2015, A\&A, 580, A17

Gilmore, G., Randich, S., Asplund, M., et al. 2012, The Messenger, 147, 25

Girardi, L., Bressan, A., Bertelli, G., \& Chiosi, C. 2000, A\&AS, 141, 371

Grand, R. J. J., Bustamante, S., Gómez, F. A., et al. 2018, MNRAS, 474, 3629

Grisoni, V., Spitoni, E., Matteucci, F., et al. 2017, MNRAS, 472, 3637

Gustafsson, B., Edvardsson, B., Eriksson, K., et al. 2008, A\&A, 486, 951

Handrich, E., Steudel, A., \& Walther, H. 1969, Phys. Lett. A, 29, 486

Haywood, M., Di Matteo, P., Lehnert, M. D., Katz, D., \& Gómez, A. 2013, A\&A, 560, A109

Heiter, U., Lind, K., Asplund, M., et al. 2015, Phys. Scr., 90, 054010

Hermann, G., Lasnitschka, G., Schwabe, C., \& Spengler, D. 1993, Spectrochim. Acta, 48, 1259

Johann, U., Dembczyński, J., \& Ertmer, W. 1981, Z. Phys. A Hadrons Nucl., 303, 7

Joshi, Y. C. 2007, MNRAS, 378, 768

Jurgenson, C., Fischer, D., McCracken, T., et al. 2016, J. Astron. Instrum., 5, 1650003

Karakas, A. I. 2010, MNRAS, 403, 1413

Katz, D., Sartoretti, P., Cropper, M., et al. 2019, A\&A, 622, A205

Kobayashi, C., \& Nakasato, N. 2011, ApJ, 729, 16

Kobayashi, C., Umeda, H., Nomoto, K., Tominaga, N., \& Ohkubo, T. 2006, ApJ, 653, 1145

Kordopatis, G., Recio-Blanco, A., de Laverny, P., et al. 2011, A\&A, 535, A107

Korotin, S., Andrievsky, S., Caffau, E., \& Bonifacio, P. 2017, in Stars: From Collapse to Collapse, eds. Y. Y. Balega, D. O. Kudryavtsev, I. I. Romanyuk, \& I. A. Yakunin, ASP Conf. Ser., 510, 141

Kurucz, R. 1993, Diatomic Molecular Data for Opacity Calculations. Kurucz CD-ROM No. 15 (Cambridge, Massachusetts: Smithsonian Astrophysical Observatory), 15 
Li, C., Zhao, G., Zhai, M., \& Jia, Y. 2018, ApJ, 860, 53

Luri, X., Brown, A. G. A., Sarro, L. M., et al. 2018, A\&A, 616, A9

Majewski, S. R., Schiavon, R. P., Frinchaboy, P. M., et al. 2017, AJ, 154, 94

Masseron, T., Plez, B., Van Eck, S., et al. 2014, A\&A, 571, A47

Michalik, D., Lindegren, L., \& Hobbs, D. 2015, A\&A, 574, A115

Miglio, A., Chiappini, C., Mosser, B., et al. 2017, Astron. Nachr., 338, 644

Mikolaitis, Š., Hill, V., Recio-Blanco, A., et al. 2014, A\&A, 572, A33

Mikolaitis, Š., de Laverny, P., Recio-Blanco, A., et al. 2017, A\&A, 600, A22

Mikolaitis, Š., Tautvaišienè, G., Drazdauskas, A., et al. 2018, PASP, 130, 074202

Mints, A., \& Hekker, S. 2017, A\&A, 604, A108

Mishenina, T. V., Gorbaneva, T. I., Basak, N. Y., Soubiran, C., \& Kovtyukh, V. V. 2011, Astron. Rep., 55, 689

Mishenina, T., Gorbaneva, T., Pignatari, M., Thielemann, F.-K., \& Korotin, S. A. 2015, MNRAS, 454, 1585

Mishenina, T., Pignatari, M., Côté, B., et al. 2017, MNRAS, 469, 4378

Mitchell, Jr., W. E., \& Mohler, O. C. 1965, ApJ, 141, 1126

Moore, C. E., Minnaert, M. G. J., \& Houtgast, J. 1966, The Solar Spectrum 2935 A to 8770 A (Washington: National Bureau of Standards Monograph)

Neves, V., Santos, N. C., Sousa, S. G., Correia, A. C. M., \& Israelian, G. 2009, A\&A, 497, 563

Nissen, P. E., Silva Aguirre, V., Christensen-Dalsgaard, J., et al. 2017, A\&A 608, A112

Nomoto, K., Kobayashi, C., \& Tominaga, N. 2013, ARA\&A, 51, 457

Prantzos, N., Abia, C., Limongi, M., Chieffi, A., \& Cristallo, S. 2018, MNRAS, 476, 3432

Ram, R. S., Brooke, J. S. A., Bernath, P. F., Sneden, C., \& Lucatello, S. 2014 ApJS, 211, 5

Rauer, H., Catala, C., Aerts, C., et al. 2014, Exp. Astron., 38, 249

Rauer, H., Aerts, C., Cabrera, J., \& Team, P. L. A. T. O. 2016, Nachr., 337, 961

Recio-Blanco, A., de Laverny, P., Kordopatis, G., et al. 2014, A\&A, 567, A5
Ricker, G. R., Winn, J. N., Vanderspek, R., et al. 2015, J. Astron. Telesc. Instrum. Syst., 1, 014003

Rojas-Arriagada, A., Recio-Blanco, A., de Laverny, P., et al. 2016, A\&A, 586, A39

Romano, D., Karakas, A. I., Tosi, M., \& Matteucci, F. 2010, A\&A, 522, A32

Saito, Y.-J., Takada-Hidai, M., Honda, S., \& Takeda, Y. 2009, PASJ, 61, 549

Schönrich, R., Binney, J., \& Dehnen, W. 2010, MNRAS, 403, 1829

Scott, P., Asplund, M., Grevesse, N., Bergemann, M., \& Sauval, A. J. 2015, A\&A, 573, A26

Sharma, S., Stello, D., Buder, S., et al. 2018, MNRAS, 473, 2004

Shi, J. R., Gehren, T., Zeng, J. L., Mashonkina, L., \& Zhao, G. 2014, ApJ, 782, 80

Skrutskie, M. F., Cutri, R. M., Stiening, R., et al. 2006, AJ, 131, 1163

Smiljanic, R., Korn, A. J., Bergemann, M., et al. 2014, A\&A, 570, A122

Sneden, C. A. 1973, PhD Thesis, The University of Texas at Austin

Sneden, C., Lucatello, S., Ram, R. S., Brooke, J. S. A., \& Bernath, P. 2014, ApJS, 214,26

Sneden, C., Cowan, J. J., Kobayashi, C., et al. 2016, ApJ, 817, 53

Spina, L., Meléndez, J., Karakas, A. I., et al. 2016, A\&A, 593, A125

Stetson, P. B., \& Pancino, E. 2008, PASP, 120, 1332

Sullivan, P. W., Winn, J. N., Berta-Thompson, Z. K., et al. 2015, ApJ, 809, 77

Takeda, Y., Zhao, G., Chen, Y.-Q., Qiu, H.-M., \& Takada-Hidai, M. 2002, PASJ, 54, 275

Takeda, Y., Kaneko, H., Matsumoto, N., et al. 2009, PASJ, 61, 563

van den Hoek, L. B., \& Groenewegen, M. A. T. 1997, A\&AS, 123, 305

Woosley, S. E., \& Weaver, T. A. 1995, ApJS, 101, 181

Yan, H. L., Shi, J. R., Nissen, P. E., \& Zhao, G. 2016, A\&A, 585, A102

Zhao, G., Mashonkina, L., Yan, H. L., et al. 2016, ApJ, 833, 225

Zucker, D. B., de Silva, G., Freeman, K., Bland-Hawthorn, J., \& Hermes Team

2012, in Galactic Archaeology: Near-Field Cosmology and the Formation of the Milky Way, eds. W. Aoki, M. Ishigaki, T. Suda, T. Tsujimoto, \& N. Arimoto, ASP Conf. Ser., 458, 421 


\section{Appendix A: Supplementary material}

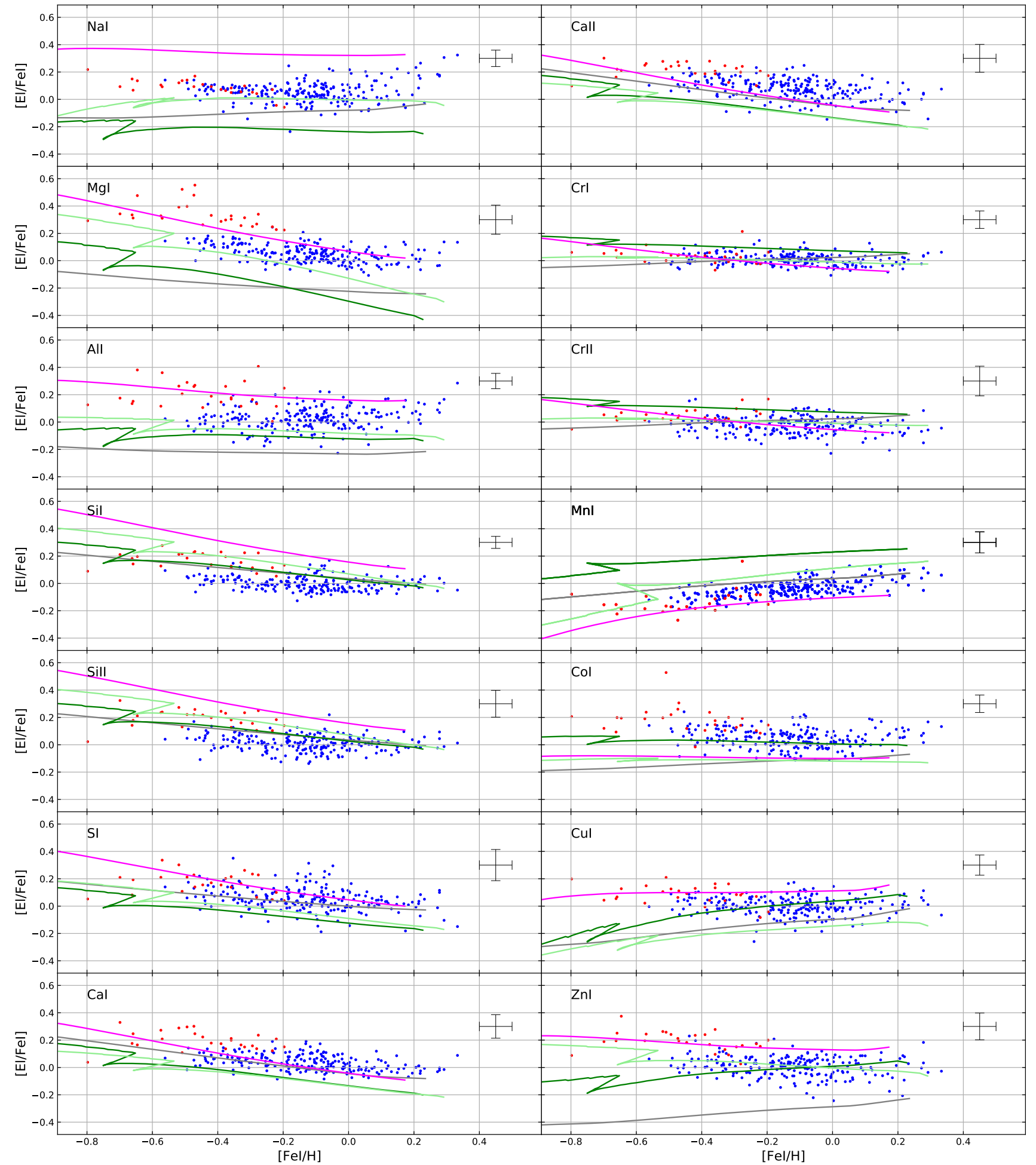

Fig. A.1. Observed element-to-iron abundance ratios as a function of the $[\mathrm{Fe} \mathrm{I} / \mathrm{H}]$ abundance. Shown are cases where some models are able to explain the data. All notations are as in Fig. 9. 


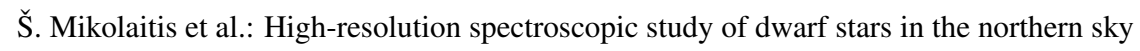

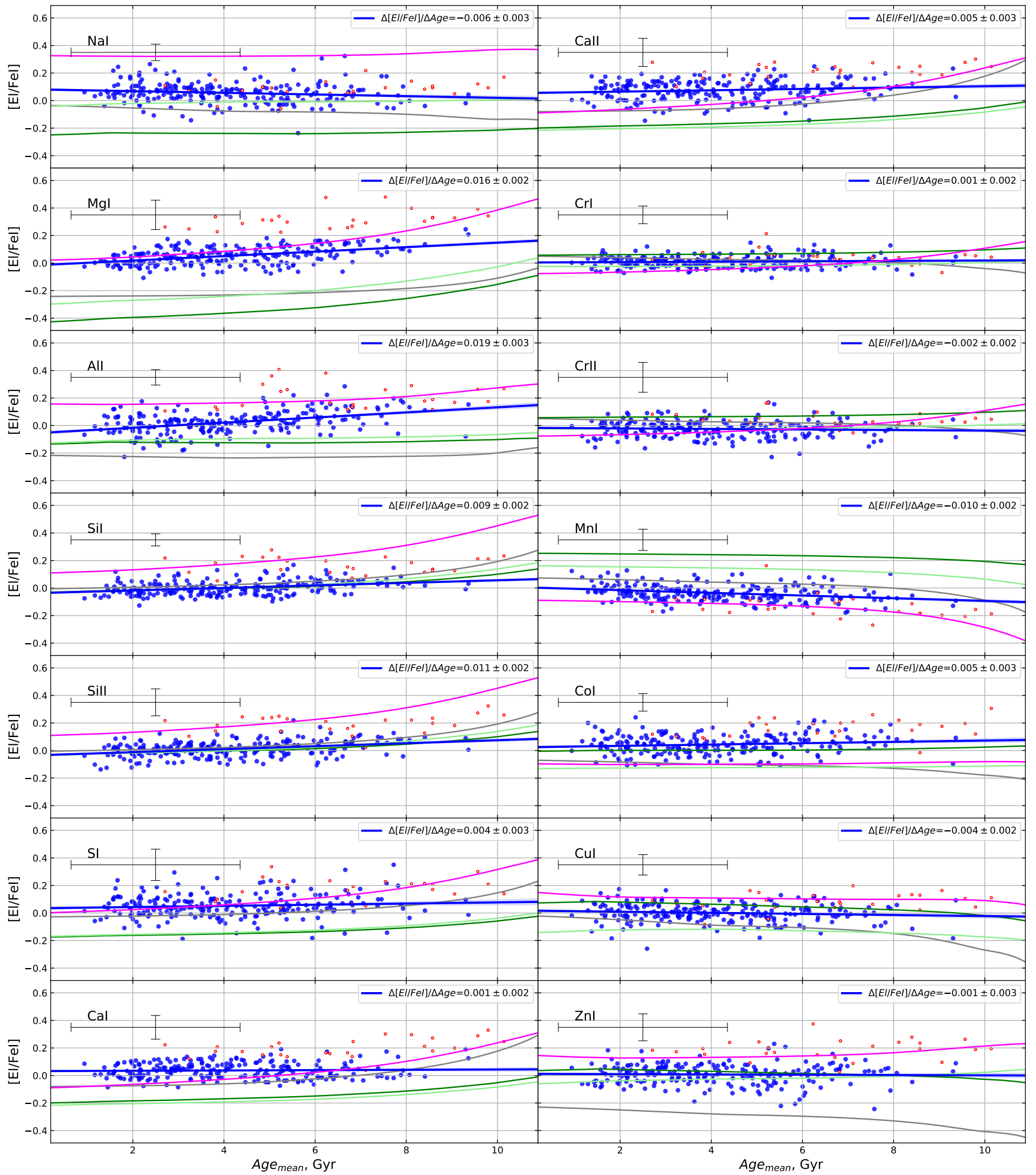

Fig. A.2. Observed element-to-iron abundance ratios as a function of the age compared to models. Shown are cases where some models are able to explain the data. All notations are as in Fig. 10. 
Table A.1. Contents of the supplementary table, which is available at the CDS.

\begin{tabular}{|c|c|c|c|}
\hline $\mathrm{Col}$ & Label & Units & Explanations \\
\hline 1 & ID & - & Tycho catalogue identification \\
\hline 2 & TESS_ID & - & ID in the TESS catalogue \\
\hline 3 & $T_{\mathrm{eff}}$ & $\mathrm{K}$ & Effective temperature \\
\hline 4 & $e_{\mathrm{Teff}}$ & $\mathrm{K}$ & Error on effective temperature \\
\hline 5 & $\log g$ & $\operatorname{dex}$ & Surface gravity \\
\hline 6 & $e \_\log g$ & dex & Error on surface gravity \\
\hline 7 & {$[\overline{\mathrm{Fe}} / \mathrm{H}]$} & - & Metallicity \\
\hline 8 & $e_{-}[\mathrm{Fe} / \mathrm{H}]$ & - & Error on metallicity \\
\hline 9 & $V_{\mathrm{t}}$ & $\mathrm{km} \mathrm{s}^{-1}$ & Microturbulence velocity \\
\hline 10 & $e_{-} \mathrm{Vt}$ & $\mathrm{km} \mathrm{s}^{-1}$ & Error on microturbulence velocity \\
\hline 11 & Vrad & $\mathrm{km} \mathrm{s}^{-1}$ & Radial velocity \\
\hline 12 & $e_{-}$Vrad & $\mathrm{km} \mathrm{s}^{-1}$ & Error on radial velocity \\
\hline 13 & Age & Gyr & Age \\
\hline 14 & $e \_$Age & Gyr & Error on age \\
\hline 15 & $\bar{U}$ & $\mathrm{~km} \mathrm{~s}^{-1}$ & $U$ velocity \\
\hline 16 & $e_{-} U$ & $\mathrm{~km} \mathrm{~s}^{-1}$ & Error on $U$ velocity \\
\hline 17 & $\bar{V}$ & $\mathrm{~km} \mathrm{~s}^{-1}$ & $V$ velocity \\
\hline 18 & $e_{-} V$ & $\mathrm{~km} \mathrm{~s}^{-1}$ & Error on $V$ velocity \\
\hline 19 & $\bar{W}$ & $\mathrm{~km} \mathrm{~s}^{-1}$ & $W$ velocity \\
\hline 20 & $e_{-} W$ & $\mathrm{~km} \mathrm{~s}^{-1}$ & Error on $W$ velocity \\
\hline 21 & $d$ & $\mathrm{kpc}$ & Distance calculated $1 / \mathrm{plx}$ \\
\hline 22 & $e \_\mathrm{d}$ & $\mathrm{kpc}$ & Error on distance \\
\hline 23 & $R_{\text {mean }}$ & $\mathrm{kpc}$ & Mean Galactocentric distance \\
\hline 24 & $e \_R_{\text {mean }}$ & $\mathrm{kpc}$ & Error on mean Galactrocentric distance \\
\hline 25 & $z_{\max }$ & $\mathrm{kpc}$ & Distance from Galactic plane \\
\hline 26 & $e \_z \max$ & $\mathrm{kpc}$ & Error on distance from Galactic plane \\
\hline 27 & $e^{-1}$ & - & Orbital eccentricity \\
\hline 28 & $e \_\mathrm{e}$ & - & Error on orbital eccentricity \\
\hline 29 & $\overline{T D} / D$ & - & Thick-to-thin disc probability ratio \\
\hline 30 & {$\left[\mathrm{Na} / / \mathrm{Fe}_{\mathrm{I}}\right]$} & - & Sodium-to-iron ratio \\
\hline 31 & $e_{-}[\mathrm{Na} \mathrm{I} / \mathrm{Fe} \mathrm{I}]$ & - & Error on sodium-to-iron ratio \\
\hline 32 & {$\left[\mathrm{Mg}_{\mathrm{I}} / \mathrm{Fe} \mathrm{I}\right]$} & - & Magnesium-to-iron ratio \\
\hline 33 & $e_{-}\left[\mathrm{Mg} \mathrm{I} / \mathrm{Fe}_{\mathrm{I}}\right]$ & - & Error on magnesium-to-iron ratio \\
\hline 34 & {$[\mathrm{Al} \mathrm{I} / \mathrm{Fe} \mathrm{I}]$} & - & Aluminium-to-iron ratio \\
\hline 35 & $e_{-}[\mathrm{Al} \mathrm{I} / \mathrm{Fe} \mathrm{I}]$ & - & Error on aluminium-to-iron ratio \\
\hline 36 & {$[\mathrm{Si}$ I/Fe $\mathrm{I}]$} & - & Silicon-to-iron ratio \\
\hline 37 & $e_{-}[\mathrm{Si} \mathrm{I} / \mathrm{Fe} \mathrm{I}]$ & - & Error on silicon-to-iron ratio \\
\hline 38 & {$\left[\overline{\mathrm{Si}}_{\mathrm{II}} / \mathrm{Fe} \mathrm{I}\right]$} & - & Ionised silicon-to-iron ratio \\
\hline 39 & $e_{-}\left[\mathrm{Si}\right.$ II $\left./ \mathrm{Fe}_{\mathrm{I}}\right]$ & - & Error on ionised silicon-to-iron ratio \\
\hline 40 & {$\left[\overline{\mathrm{S}}_{\mathrm{I}} / \mathrm{Fe} \mathrm{I}\right]$} & - & Sulphur-to-iron ratio \\
\hline 41 & $e_{-}\left[\mathrm{S}_{\mathrm{I}} / \mathrm{Fe}_{\mathrm{I}}\right]$ & - & Error on sulphur-to-iron ratio \\
\hline 42 & {$\left[\overline{\mathrm{K}}_{\mathrm{I}} / \mathrm{Fe}_{\mathrm{I}}\right]$} & - & Potassium-to-iron ratio \\
\hline 43 & $e_{-}\left[\mathrm{K}_{\mathrm{I}} / \mathrm{Fe}_{\mathrm{I}}\right]$ & - & Error on potassium-to-iron ratio \\
\hline 44 & {$[\overline{\mathrm{Ca}} \mathrm{I} / \mathrm{Fe} \mathrm{I}]$} & - & Calcium-to-iron ratio \\
\hline 45 & $e_{-}[\mathrm{Ca} \mathrm{I} / \mathrm{Fe} \mathrm{I}]$ & - & Error on calcium-to-iron ratio \\
\hline 46 & {$[\overline{\mathrm{Ca}} \mathrm{II} / \mathrm{Fe} \mathrm{I}]$} & - & Ionised calcium-to-iron ratio \\
\hline 47 & $e_{-}[\mathrm{Ca} \mathrm{II} / \mathrm{Fe} \mathrm{I}]$ & - & Error on ionised calcium-to-iron ratio \\
\hline 48 & {$\left[\mathrm{Sc} \mathrm{I} / \mathrm{Fe}_{\mathrm{I}}\right]$} & - & Scandium-to-iron ratio \\
\hline 49 & $e_{-}[\mathrm{Sc} \mathrm{I} / \mathrm{Fe} \mathrm{I}]$ & - & Error on scandium-to-iron ratio \\
\hline 50 & {$\left[\mathrm{Sc}\right.$ II $\left./ \mathrm{Fe} \mathrm{I}_{\mathrm{I}}\right]$} & - & Ionised scandium-to-iron ratio \\
\hline 51 & $e_{-}[\mathrm{Sc} \mathrm{II} / \mathrm{Fe} \mathrm{I}]$ & - & Error on ionised scandium-to-iron ratio \\
\hline 52 & {$[\mathrm{Ti} \mathrm{I} / \mathrm{Fe} \mathrm{I}]$} & - & Titanium-to-iron ratio \\
\hline 53 & $e_{-}[\mathrm{Ti} \mathrm{I} / \mathrm{Fe} \mathrm{I}]$ & - & Error on titanium-to-iron ratio \\
\hline 54 & {$\left[\mathrm{Ti}\right.$ II $\left./ \mathrm{Fe}_{\mathrm{I}}\right]$} & - & Ionised titanium-to-iron ratio \\
\hline
\end{tabular}


Table A.1. continued.

\begin{tabular}{|c|c|c|c|}
\hline Col & Label & Units & Explanations \\
\hline 55 & $e_{-}[\mathrm{Ti} \mathrm{II} / \mathrm{Fe} \mathrm{I}]$ & - & Error on ionised titanium-to-iron ratio \\
\hline 56 & {$\left[\overline{\mathrm{V}}_{\mathrm{I}} / \mathrm{Fe}_{\mathrm{I}}\right]$} & - & Vanadium-to-iron ratio \\
\hline 57 & $e_{-}\left[\mathrm{V}_{\mathrm{I}} / \mathrm{Fe}_{\mathrm{I}}\right]$ & - & Error on vanadium-to-iron ratio \\
\hline 58 & {$\left[\mathrm{Cr} \mathrm{I} / \mathrm{Fe}_{\mathrm{I}}\right]$} & - & Chromium-to-iron ratio \\
\hline 59 & $e_{-}[\mathrm{Cr} \mathrm{I} / \mathrm{Fe} \mathrm{I}]$ & - & Error on chromium-to-iron ratio \\
\hline 60 & {$\left[\mathrm{Cr}_{\mathrm{II}} / \mathrm{Fe}_{\mathrm{I}}\right]$} & - & Ionised chromium-to-iron ratio \\
\hline 61 & $e_{-}[\mathrm{Cr} \mathrm{II} / \mathrm{Fe} \mathrm{I}]$ & - & Error on ionised chromium-to-iron ratio \\
\hline 62 & {$\left[\mathrm{Mn} \mathrm{I} / \mathrm{Fe}_{\mathrm{I}}\right]$} & - & Manganese-to-iron ratio \\
\hline 63 & $e_{-}\left[\mathrm{Mn}_{\mathrm{I}} / \mathrm{Fe}_{\mathrm{I}}\right]$ & - & Error on manganese-to-iron ratio \\
\hline 64 & {$[\mathrm{Co} \mathrm{I} / \mathrm{Fe} \mathrm{I}]$} & - & Cobalt-to-iron ratio \\
\hline 65 & $e_{-}\left[\mathrm{Co} \mathrm{I} / \mathrm{Fe}_{\mathrm{I}}\right]$ & - & Error on cobalt-to-iron ratio \\
\hline 66 & {$[\mathrm{Ni}$ I/Fe I $]$} & - & Nickel-to-iron ratio \\
\hline 67 & $e_{-}[\mathrm{Ni} \mathrm{I} / \mathrm{Fe} \mathrm{I}]$ & - & Error on nickel-to-iron ratio \\
\hline 68 & {$\left[\mathrm{Cu} / / \mathrm{Fe}_{\mathrm{I}}\right]$} & - & Copper-to-iron ratio \\
\hline 69 & $e_{-}\left[\mathrm{Cu} \mathrm{I} / \mathrm{Fe}_{\mathrm{I}}\right]$ & - & Error on copper-to-iron ratio \\
\hline 70 & {$\left[\mathrm{Zn} \mathrm{I} / \mathrm{Fe}_{\mathrm{I}}\right]$} & - & Zinc-to-iron ratio \\
\hline 71 & $e_{-}[\mathrm{Zn} \mathrm{I} / \mathrm{Fe} \mathrm{I}]$ & - & Error on zinc-to-iron ratio \\
\hline 72 & {$[\mathrm{Fe} \mathrm{I} / \mathrm{H}]$} & - & Iron abundance \\
\hline 73 & $e \_[\mathrm{Fe} \mathrm{I} / \mathrm{H}]$ & - & Error on iron abundance \\
\hline 74 & {$[\mathrm{Fe} I \mathrm{II} / \mathrm{H}]$} & - & Ionised iron abuncance \\
\hline 75 & $e_{-}[\mathrm{Fe}$ II $/ \mathrm{H}]$ & - & Error on ionised iron abundance \\
\hline
\end{tabular}

